Ann. Zootech., 1983, 32 (2), 215-246

\title{
Comparaison de différentes méthodes d'évaluation du coefficient d'utilisation digestive des aliments concentrés par le ruminant
}

\author{
Sylvie GIGER et D. SAUVANT \\ I.N.R.A., Laboratoire de Recherches de la Chaire de Zootechnie, \\ I.N.A. Paris-Grignon, \\ 16, rue Claude-Bernard, $F 75231$ Paris Cedex 05
}

\begin{abstract}
Résumé
Le coefficient d'utilisation digestive (CUD) d'un aliment concentré pour ruminant ne peut être estimé directement. Différentes méthodes d'évaluation de ce CUD ont été proposées, et l'objet du présent travail est de réaliser une comparaison systématique de ces méthodes. Les données utilisées comme base concernent un ensemble de mesures effectuées sur seize paramètres avec quatre régimes mixtes différents (un aliment concentré associé à un fourrage).

La méthode classique dite "des différences» donne des résultats entachés d'une forte incertitude quand la moyenne du pourcentage du principe apporté par l'aliment concentré est inférieure à $20 \mathrm{p}$. 100 . L'utilisation d'un système de pondération ne modifie pas statistiquement les valeurs obtenues, mais permet de diminuer les incertitudes. Des conclusions comparables sont obtenues quand les données enregistrées individuellement pour le foin sont prises en considération à la place d'une valeur moyenne (méthode des différences à fourrage variable).
\end{abstract}

Avec la méthode des équations, il existe une valeur minimale de l'incertitude qui est fonction du niveau de troncage (écart minimum entre les deux pourcentages d'un constituant donné apporté par le foin ou le concentré) et du nombre de données ainsi retenu. Le niveau moyen du troncage observé a été d'environ 10 p. 100.

Un modèle de régression progressive incluant le pourcentage de constituant apporté par le concentré a également été utilisé, permettant ainsi de tester d'éventuels phénomènes liés aux interactions digestives ou au niveau alimentaire.

Les résultats obtenus par ces deux dernières méthodes sont très corrélés et varient quelque peu de ceux obtenus par la méthode des différences.

De la comparaison de ces trois types de méthodes, il ressort que les incertitudes obtenues sont fonction, non seulement des pourcentages moyens du principe apporté par le concentré, mais également de la répartition de ces pourcentages. Seule, la méthode des régressions permet en général de mettre en évidence la présence d'un éventuel phénomène d'interaction digestive.

Cette étude montre, en outre, qu'il est important de connaître l'incertitude attachée à un coefficient de digestibilité ainsi que la méthode employée pour le déterminer. 


\section{Introduction}

Les rations distribuées aux animaux ruminants comprennent un ou plusieurs aliments grossiers qui doivent éventuellement être complétés par un ou plusieurs aliments concentrés riches en énergie et, le cas échéant, en tout autre élément nutritif pour permettre à ces animaux d'extérioriser leur potentiel de production. Un problème important posé par l'emploi de telles rations mixtes réside dans le fait que leur valeur nutritive énergétique est, pour des raisons d'interaction digestive dans l'ensemble « rumen + réseau», différente de celle que l'on pourrait prévoir en se basant sur la connaissance des valeurs énergétiques de ces constituants, pris séparément.

La présence de ces phénomènes d'interaction digestive qui ont été mis en évidence depuis longtemps (MitchelL, 1937), rend quasiment impossible une estimation précise des coefficients d'utilisation digestive (CUD) et donc, de la valeur nutritive des aliments concentrés puisque, notamment pour éviter des problèmes de pathologie digestive ou d'appétence, il n'est pas possible d'utiliser une ration entièrement composée de concentrés.

Différentes méthodes, dont celles décrites par Schneider \& FlatT (1975), peuvent être appliquées pour estimer le CUD d'un des composants d'un aliment concentré. Charlet-Lery \& Leroy (1955) et Frederiksen (1973) ont effectué des comparaisons entre certaines de ces méthodes ; par contre, à notre connaissance, aucune comparaison systématique n’a été entreprise jusqu’à présent. Il était donc nécessaire de comparer, à partir de plusieurs séries de mesures de coefficients de digestibilité de fourrages et de rations mixtes, les différentes méthodes applicables pour estimer les CUD des composants des aliments concentrés et pour tenter de mettre en évidence leurs avantages et inconvénients respectifs. Cette démarche constitue une étape indispensable de la mise au point d'une méthode normalisée d'évaluation des coefficients de digestibilité des composants des aliments concentrés distribués aux animaux ruminants.

\section{Matériel et méthodes}

\section{A. Protocoles expérimentaux}

Les résultats utilisés dans ce travail sont issus de deux séries d'expérimentation conduites chacune sur trois couples de chèvres de race Alpine, en lactation. Les chèvres d'un même couple se différencient par le type d'aliment concentré. Les protocoles expérimentaux qui ont permis de tester quatre aliments composés ont été décrits par ailleurs (Giger et al., 1983 a et b) et sont rappelés dans le tableau 1. La composition analytique des aliments expérimentaux est reportée au tableau 2.

Les périodes de mesure de digestibilité interviennent après un minimum de trois semaines d'adaptation au régime proposé. Les valeurs des ingesta et d'excreta correspondent aux moyennes de mesures journalières effectuées sur cinq jours consécutifs. Dans le cas des fourrages, des analyses séparées de l'ingéré et de refus individuels ont été effectuées. 


\section{TABLEAU 1}

Protocoles expérimentaux.

Plan of the experiments.

\begin{tabular}{|c|c|c|}
\hline & $\begin{array}{c}\text { Expérience } \mathrm{n}^{\circ} 1 \\
\text { Experiment } 1\end{array}$ & $\begin{array}{c}\text { Expérience } \mathrm{n}^{0} 2 \\
\text { Experiment } 2\end{array}$ \\
\hline $\begin{array}{l}\text { Nombre d'animaux } \ldots \ldots \ldots \\
\text { Number of animals }\end{array}$ & 6 & 6 \\
\hline $\begin{array}{l}\text { Stade de lactation au début de } \\
\text { l'expérience .............. } \\
\text { Stage of lactation at the be- } \\
\text { ginning of the experiment }\end{array}$ & $\begin{array}{l}17^{\mathrm{e}} \text { semaine après mise bas } \\
17 \text { th week after parturition }\end{array}$ & $\begin{array}{l}13^{\mathrm{e}} \text { semaine après mise bas } \\
13 \text { th week after parturition }\end{array}$ \\
\hline $\begin{array}{l}\text { Durée totale de l'expérience } \\
\text { Total duration of the experi- } \\
\text { ment }\end{array}$ & $\begin{array}{l}14 \text { semaines } \\
14 \text { weeks }\end{array}$ & $\begin{array}{l}13 \text { semaines } \\
13 \text { weeks }\end{array}$ \\
\hline $\begin{array}{l}\text { Type de ration } \\
\text { Kind of diet }\end{array}$ & & \\
\hline $\begin{array}{l}\text { 1. Ration mixte } \\
\text { Mixed diet }\end{array}$ & & \\
\hline $\begin{array}{l}\text { Foin de luzerne } \ldots \ldots \ldots \\
\text { Lucerne hay }\end{array}$ & $\begin{array}{l}1150 \mathrm{~g} \mathrm{MS} / \mathrm{j} \\
1150 \mathrm{~g} \mathrm{DM} / \mathrm{d}\end{array}$ & $\begin{array}{l}\text { ad libitum (15 p. } 100 \text { refus } \\
\text { toléré) } \\
\text { ad libitum (level of relusal : } \\
15 \text { p. 100) }\end{array}$ \\
\hline $\begin{array}{l}\text { Aliment concentré } \ldots \ldots \ldots \\
\text { Concentrate }\end{array}$ & $\begin{array}{l}310 \mathrm{~g} \mathrm{MS} / \mathrm{kg} \text { lait } \\
\text { à } 35 \mathrm{p} .1000 \\
310 \mathrm{~g} \mathrm{DM} / F C M \\
(35 \mathrm{p.} 1000) \\
\text { pendant } 10 \text { semaines dont } 6 \\
\text { de contrôle } \\
\text { for ten weeks (including } 6 \\
\text { control weeks) }\end{array}$ & $\begin{array}{l}350 \mathrm{~g} \mathrm{MS} / \mathrm{kg} \text { lait } \\
\text { à } 35 \mathrm{p} .1000 \\
350 \mathrm{~g} \mathrm{DM} / \mathrm{kg} \mathrm{FCM} \\
(35 \mathrm{p} .1000) \text { for } \\
\text { pendant } 5 \mathrm{semaines} \mathrm{dont} 2 \\
\text { de contrôle } \\
\text { for } 5 \text { weeks (including } \\
2 \text { control weeks) } \\
490 \mathrm{~g} \mathrm{MS} / \mathrm{kg} \text { lait } \\
\text { à } 35 \mathrm{p.} 1000 \\
\text { pendant } 4 \text { semaines dont } 2 \\
\text { de contrôle } \\
490 \mathrm{~g} \mathrm{DM} / \mathrm{kg} \text { FCM } \\
(35 \mathrm{p} .1000) \\
\text { four weeks (including } 2 \\
\text { control weeks) }\end{array}$ \\
\hline $\begin{array}{l}\text { 2. Fourrage seul } \\
\text { Forage alone }\end{array}$ & & \\
\hline $\begin{array}{l}\text { Foin de luzerne } \ldots \ldots \ldots \\
\text { Lucerne hay }\end{array}$ & $\begin{array}{l}\text { ad libitum } \\
(15 \text { p. } 100 \text { de refus toléré) } \\
\text { (level of refusal : } 15 \text { p. 100) } \\
\text { pendant } 4 \text { semaines dont } 2 \\
\text { de contrôle } \\
\text { for } 4 \text { weeks (including } 2 \\
\text { control weeks) }\end{array}$ & $\begin{array}{l}\text { ad libitum } \\
(15 \mathrm{p} .100 \text { de refus toléré) } \\
\text { (level of refusal }: 15 \text { p. } 100) \\
\text { pendant } 4 \text { semaines dont } 2 \\
\text { de contrôle } \\
\text { for } 4 \text { weeks (including } 2 \\
\text { control weeks) }\end{array}$ \\
\hline
\end{tabular}


TABLeau 2

Composition chimique et valeur énergétique des différents aliments.

Chemical composition and nutritive value of the different feeds used.

\begin{tabular}{|c|c|c|c|c|c|c|}
\hline \multirow{2}{*}{$\begin{array}{l}\text { p. } 100 \mathrm{MS} \\
\text { p. } 100 \mathrm{DM}\end{array}$} & \multicolumn{3}{|c|}{$\begin{array}{c}\text { Expérierce } \mathrm{n}^{\circ} 1 \\
\text { Experiment } 1\end{array}$} & \multicolumn{3}{|c|}{$\begin{array}{c}\text { Expérience } \mathrm{n}^{0} 2 \\
\text { Experiment } 2\end{array}$} \\
\hline & $\begin{array}{c}\text { Concentré } 1 \\
\text { Concen- } \\
\text { trate } 1 \\
\end{array}$ & $\begin{array}{c}\text { Concentré } 2 \\
\text { Concen- } \\
\text { trate } 2\end{array}$ & $\begin{array}{l}\text { Foin } \\
\text { Hay }\end{array}$ & $\begin{array}{l}\text { Concentré } 1 \\
\text { Concen- } \\
\text { trate } 1 \\
\end{array}$ & $\begin{array}{c}\text { Concentré } 2 \\
\text { Concen- } \\
\text { trate } 2\end{array}$ & $\begin{array}{l}\text { Foin } \\
\text { Hay }\end{array}$ \\
\hline & $P$ & $\mathbf{R}$ & & A & $\mathbf{M}$ & \\
\hline $\begin{array}{l}\text { Cellulose brute } \ldots \\
\text { Crude fibre }\end{array}$ & 10,0 & 11,8 & 32,4 & 8,4 & 17,4 & 30,5 \\
\hline $\begin{array}{l}\text { Matières azotées } \\
\text { totales } \ldots \ldots \ldots \ldots \ldots \\
\text { Crude protein }\end{array}$ & 21,3 & 20,0 & 17,2 & 20,5 & 16,6 & 18,5 \\
\hline $\begin{array}{l}\text { Extrait etheré } \ldots \ldots \\
\text { Ether extract }\end{array}$ & 3,3 & 2,0 & - & 3,0 & 1,2 & - \\
\hline $\begin{array}{l}\text { Matières minérales } \\
\text { Ash }\end{array}$ & 9,7 & 8,0 & 8,5 & 7,4 & 8,6 & 10,0 \\
\hline $\begin{array}{l}\text { NDF } \\
N D F\end{array}$ & 37,8 & 31,5 & 49,2 & 21,2 & 51,5 & 48,3 \\
\hline $\begin{array}{l}\mathrm{ADF} \\
A D F\end{array}$ & 14,2 & 14,8 & 34,8 & 9,6 & 25,8 & 33,3 \\
\hline $\begin{array}{l}\text { Lignine } \\
\text { Lignin }\end{array}$ & 3,7 & 1,3 & 8,0 & 1,0 & 3,8 & 7,5 \\
\hline $\begin{array}{l}\text { Valeur nutritive (1) } \\
\text { (UFL/kg MS) } \\
\text { Nutritive value } \\
\text { (Milk } F U / k g D M)\end{array}$ & 0,96 & 1,06 & 0,68 & 1,01 & 1,01 & 0,70 \\
\hline $\begin{array}{l}\text { Digestibilité de la } \\
\text { matière organique } \\
\text { (DMO) (1) ........ } \\
\text { Organic matter diges- } \\
\text { tibility (OMD) (1) }\end{array}$ & 76,4 & 83,3 & 60,6 & 81,3 & 79,5 & 62,4 \\
\hline
\end{tabular}

(1) Estimée in vivo par la méthode des régressions pour les aliments concentrés - Estimated in vivo by the regression method for the concentrates.

Pour chaque période de bilan et pour chaque chèvre, les CUD de seize principes ont été calculés. Les principes choisis ainsi que les abréviations qui seront utilisées dans le texte sont récapitulés dans le tableau 3. 
TableaU 3

Liste et abréviations des principes retenus pour les calculs de digestibilité.

List and abbreviations of nutrients used for the digestibility measurements.

\begin{tabular}{|c|c|c|}
\hline $\begin{array}{l}\text { Nom du principe } \\
\text { Name }\end{array}$ & $\begin{array}{l}\text { Abréviation } \\
\text { Abbreviation }\end{array}$ & $\begin{array}{l}\text { Méthode de détermination } \\
\text { ou de calcul } \\
\text { Chemical or mathematical } \\
\text { method used }\end{array}$ \\
\hline $\begin{array}{l}\text { Matière sèche } \ldots \ldots \cdots \cdots \cdots \cdots \cdots \\
\text { Dry matter }\end{array}$ & MS & \\
\hline $\begin{array}{l}\text { Matière organique } \ldots \ldots \ldots \ldots \ldots \\
\text { Organic matter }\end{array}$ & MO & \\
\hline $\begin{array}{l}\text { Matières azotées totales } \ldots \\
\text { Crude protein }\end{array}$ & MAT & \\
\hline $\begin{array}{l}\text { Cellulose brute } \ldots \ldots \ldots \ldots \ldots \ldots \\
\text { Crude fibre }\end{array}$ & $\mathrm{CB}$ & \\
\hline $\begin{array}{l}\text { "Paroi végétale } » \ldots \ldots \ldots \ldots \ldots \\
\text { Cell-wall }\end{array}$ & NDF & $\begin{array}{l}\text { Détermination par la méthode de vaN } \\
\text { SoEST \& WINE (1967) modifiée par } \\
\text { GIGER et al. (à paraître) } \\
\text { Method of VAN SOEST \& WINE (1967) } \\
\text { modified by GIGER et al. (to be pu- } \\
\text { blished) }\end{array}$ \\
\hline $\begin{array}{l}\text { Lignocellulose } » ~ \\
\text { Lignocellulose }\end{array}$ & ADF & VAN SOEST (1963) \\
\hline $\begin{array}{l}\text { "Hémicellulose } » \ldots \ldots \ldots \ldots \ldots \ldots \\
\text { Hemicellulose }\end{array}$ & HEMI & NDF-ADF \\
\hline $\begin{array}{l}\text { Cellulose } » \ldots \ldots \ldots \ldots \ldots \ldots \ldots \\
\text { Cellulose }\end{array}$ & CELL. & ADF-ADL \\
\hline $\begin{array}{l}\text { Lignine } \\
\text { Lignin }\end{array}$ & $\mathrm{Li}$ & VAN SOEST (1963) \\
\hline $\begin{array}{l}\text { Cellulose brute soluble } . . . \ldots \ldots \ldots \\
\text { Soluble crude fibre }\end{array}$ & CBS & MO-CB \\
\hline $\begin{array}{l}\text { Paroi végétale soluble } \ldots \ldots \ldots \ldots \\
\text { Soluble cell-wall }\end{array}$ & NDS & MO-NDF \\
\hline $\begin{array}{l}\text { Lignocellulose soluble } \ldots \ldots \ldots \ldots \ldots \\
\text { Soluble lignocellulose }\end{array}$ & ADS & MO-ADF \\
\hline $\begin{array}{l}\text { Lignine soluble } \ldots \ldots \ldots \ldots \ldots \ldots \\
\text { Soluble lignin }\end{array}$ & LiS & MO-Li \\
\hline $\begin{array}{c}\text { "Holocellulose } » \ldots \ldots \ldots \ldots \ldots \ldots \\
\text { Holocellulose }\end{array}$ & HOLO & NDF-Li \\
\hline $\begin{array}{l}\text { Extractif non azoté }+ \text { extrait éthéré } \\
\text { Nitrogen free extract }+ \text { ether extract }\end{array}$ & $\mathrm{ENA}+\mathrm{EE}$ & MO-CB-MAT \\
\hline $\begin{array}{l}\text { Extractif non azoté vaN SoEST } \ldots \\
\text { VAN SOEST nitrogen free extract }\end{array}$ & NDS-MAT & MO-NDF-MAT \\
\hline
\end{tabular}




\section{B. Méthodes de calcul de la digestibilité}

\section{Principe de base des calculs}

Les mesures effectuées permettent de connaître, pour chaque ration (r) et chaque période, la quantité apparemment digérée de chaque principe alimentaire considéré, par exemple la matière organique : MO.Dig.r. Cette quantité représente la somme des éléments correspondants du foin (MO.Dig.f) et de l'aliment concentré (MO.Dig.c) : MO.Dig.r = MO.Dig.f + MO.Dig.c.

Cette équation peut s'exprimer en faisant apparaître les coefficients d'utilisation digestive du composant considéré pour la ration, le foin et l'aliment concentré : respectivement CUD.r, CUD.f et CUD.c :

$$
\text { (MO.r) (CUD.r) }=(\text { MO.f) }(\text { CUD.f })+(\text { MO.c) }(\text { CUD.c })
$$

$\mathrm{Si} \mathrm{F}$ et $\mathrm{C}$ désignent les proportions de la matière organique de la ration apportées respectivement par le foin et le concentré, l'équation devient :

$$
\text { CUD.r }=\text { F (CUD.f) }+ \text { C (CUD.c) }
$$

Cette démarche constitue la première phase commune aux trois méthodes décrites par la suite. Elle suppose implicitement que les CUD du foin et du concentré (CUD.f et CUD.c) restent constants, quelles que soient les valeurs de $F$ et de $C$. Ceci revient à faire l'hypothèse d'une additivité parfaite entre les phénomènes digestifs relatifs au foin et à l'aliment concentré. Dans le cas contraire, il y a une interaction digestive (I), parfois désignée par le terme d'associativité (1). La digestibilité de la ration ne peut alors plus être considérée comme une combinaison linéaire de celles de ses deux constituants :

$$
\text { CUD. } r=F(\text { CUD.f })+C \text { (CUD.c) }+I
$$

L'interaction digestive peut être négative $(I<0)$ ou positive $(I>0)$ comme le montre la figure 1 . La première situation se rencontre notamment lors des variations importantes de l'équilibre entre les substrats glucidiques (équilibre amidon-paroi); la seconde s'observe en particulier lorsque l'on complémente un fourrage pauvre en énergie et en azote par un aliment concentré, vecteur d'azote fermentescible.

\section{Les méthodes des différences}

Une de ces méthodes, la plus simple et la plus souvent appliquée, a servi de base à l'établissement des différentes tables de référence de la valeur nutritive des aliments concentrés. Les coefficients de digestibilité des différents principes du fourrage sont déterminés chez des animaux qui l'ingèrent comme ration unique. L'application des valeurs de CUD.r, CUD.f, $F$ et $C$ dans l'équation (1) permet de calculer le CUD du principe correspondant de l'aliment concentré.

$$
\text { CUD.c }=\frac{\text { CUD.r }-F(C U D . f)}{C}
$$

(1) Le terme «digestibilité associative » parfois utilisé dans la langue française, provient de l'adoption du mot anglais «associative digestibility ». Cette expression ne peut en fait correspondre à la définition française de l'associativité. Nous préférons l'emploi de l'expression \& digestibilité interactive » à la place de celle de «digestibilité associative ». L'interaction est, en effet, le terme utilisé pour exprimer une absence d'additivité entre plusieurs effets. 


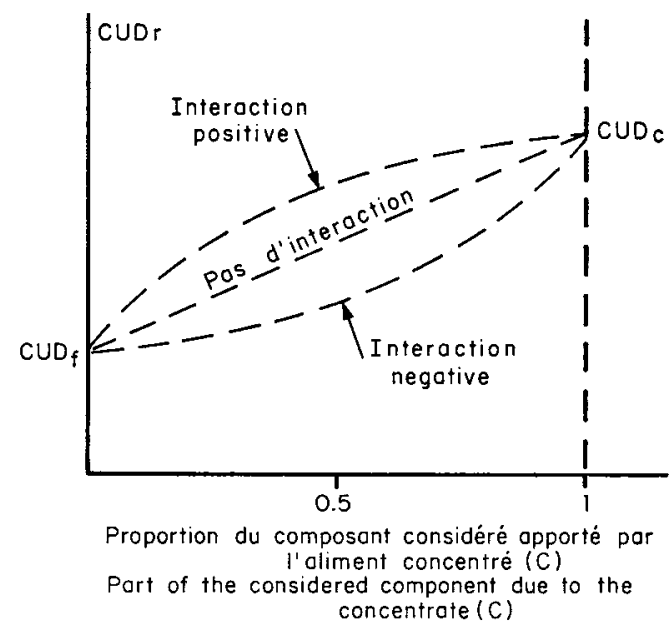

FIG. 1

Forme des courbes de CUD de la ration totale en présence ou absence d'interactions digestives.

Alternative shapes of diet digestibility curves in the presence or absence of associative digestibility.

(1) r, f, c : Voir le texte - See the text.

Dans le cas de cette méthode classique des différences, les CUD du fourrage (CUD.f) sont considérés comme étant une valeur certaine; en conséquence, l'écarttype $(\sigma)$ qui s'attache au coefficient de digestibilité de chacun des composants du concentré s'exprime de la façon suivante :

$$
\sigma(\text { CUD.c })=\frac{1}{\mathrm{C}} \sigma(\mathrm{CUD} . \mathrm{r})
$$

Ainsi, l'incertitude de la mesure de CUD.c est, d'une part, une fonction inverse de la proportion du composant considéré apporté par le concentré et, d'autre part, une fonction directe de $\sigma$ (CUD.r). Pour une valeur donnée de C, la valeur de $\sigma$ (CUD.r) dépend des variations individuelles ou analytiques.

Comme cela a été montré par Frederiksen (1973), les interactions digestives négatives ou positives, aboutissent respectivement à des sous-estimations ou à des surestimations d'autant plus importantes que, l'aliment concentré étant utilisé en quantité relativement faible, les valeurs de $\mathrm{C}$ attachées à chacun de ses composants sont faibles.

D'après l'équation (2) et du fait que plusieurs bilans ont été effectués pour des valeurs différentes de $\mathrm{C}$, il semble normal de ne pas faire la moyenne des différentes valeurs CUD.ci obtenues pour CUD.c, mais de pondérer chacune de ces valeurs par le degré de confiance qu'il convient de leur accorder. En conséquence, nous proposons d'utiliser le système de pondération suivant : 


$$
\operatorname{CUD}_{c}=\frac{\sum_{i=1}^{n} C_{c i} \operatorname{CUD}_{\mathrm{ci}}}{\sum_{i=1}^{n} \mathrm{C}_{\mathrm{i}}}
$$

et de dénommer ce mode de calcul méthode des différences pondérées.

Pour mieux cerner l'incertitude qui s'attache au résultat, il est en outre possible de combiner toute valeur mesurée de la digestibilité de la rat on aux différentes valeurs obtenues pour le fourrage distribué seul, ce qui tient compte du fait que le CUD du fourrage n'est pas une valeur certaine. Cette démarche sera désignée par le terme de «méthode des différences avec fourrage variable». Elle peut en outre être combinée à la méthode des différences pondérées.

En conséquence, sous le terme de méthode des différences, il convient de distinguer a priori quatre approches :

- la méthode classique ;

- la méthode pondérée ;

- la méthode à fourrage variable ;

- la méthode pondérée à fourrage variable.

\section{Méthode par résolution de systèmes de deux équations}

Cette méthode, décrite en particulier par von KNIERIem (1900) et Gasnier \& VACHEL (1952), ne nécessite pas d'avoir à effectuer de mesures de digestibilité de l'aliment grossier distribué seul; par contre, elle nécessite de disposer de deux mesures de digestibilité effectuées avec des proportions différentes d'aliments grossiers $\left(F_{1}\right.$ et $\left.F_{2}\right)$ et concentrés $\left(\mathrm{C}_{1}\right.$ et $\left.\mathrm{C}_{2}\right)$ afin de pouvoir réaliser un système de deux équations à deux inconnues à partir de l'équation (1) :

$$
\begin{aligned}
& \text { CUD. } r_{1}=F_{1} \text { (CUD.f) }+C_{1} \text { (CUD.c) } \\
& \text { CUD. } r_{2}=F_{2} \text { (CUD.f) }+C_{2} \text { (CUD.c) }
\end{aligned}
$$

La résolution de ce système aboutit à l'estimation pour chacun des principes des coefficients de CUD.f et CUD.c :

$$
\begin{aligned}
& \text { CUD.c }=\frac{F_{1}\left(\text { CUD. } r_{2}\right)-F_{2}\left(\text { CUD. } r_{1}\right)}{F_{1} C_{2}-F_{2} C_{1}} \\
& \text { CUD.f }=\frac{C_{1}\left(\text { CUD. } r_{2}\right)-C_{2}\left(C U D . r_{1}\right)}{F_{1} C_{2}-F_{2} C_{1}}
\end{aligned}
$$

et puisque $\mathrm{F}_{1}+\mathrm{C}_{1}=\mathrm{F}_{2}+\mathrm{C}_{2}=1$ :

$$
\begin{aligned}
& \text { CUD.c }=\frac{F_{1}\left(\text { CUD } . r_{2}\right)-F_{2}\left(C U D . r_{1}\right)}{C_{2}-C_{1}} \\
& \text { CUD.f }=\frac{C_{1}\left(\text { CUD. } r_{2}\right)-C_{2}\left(\text { CUD. } r_{1}\right)}{C_{2}-C_{1}}
\end{aligned}
$$


Cette démarche peut s'appliquer à tout couple de mesures; en conséquence, l'utilisation de plusieurs animaux considérés à différentes périodes et recevant des proportions variables d'aliments concentrés, aboutit rapidement à l'accumulation d'un nombre important d'estimations de CUD.c et de CUD.f. 11 importe alors de savoir si toutes les données ainsi obtenues doivent être prises en compte dans le calcul d'un coefficient de digestibilité.

Les variables CUD. $r_{1}$ et CUD. $r_{2}$ étant indépendantes, il est possible de calculer une estimation de l'écart-type des coefficients de digestibilité :

$$
\begin{aligned}
\sigma(\text { CUD.c }) & =\sqrt{\left(\frac{\mathrm{F}_{1}}{\mathrm{C}_{2}-\mathrm{C}_{1}}\right)^{2} \sigma^{2}\left(\mathrm{CUD} . \mathrm{r}_{2}\right)+\left(\frac{\mathrm{F}_{2}}{\mathrm{C}_{2}-\mathrm{C}_{1}}\right)^{2} \sigma^{2}\left(\text { CUD. } \mathrm{r}_{1}\right)} \\
& =\sigma \text { (CUD.r) } \sqrt{\frac{\mathrm{F}_{1}{ }^{2}+\mathrm{F}_{2}{ }^{2}}{\left(\mathrm{C}_{2}-\mathrm{C}_{1}\right)^{2}}}
\end{aligned}
$$

De même :

$$
\sigma(\text { CUD.f })=\sigma(\text { CUD.r }) \quad \sqrt{\frac{\mathrm{C}_{1}^{2}+\mathrm{C}_{2}{ }^{2}}{\left(\mathrm{C}_{2}-\mathrm{C}_{1}\right)^{2}}}
$$

L'incertitude sur l'estimation du CUD d'un composant du concentré est donc d'autant plus faible que les proportions $C_{1}$ et $C_{2 .}$ sont élevées et que l'écart de proportion $\left(C_{2}-C_{1}\right)$ entre les deux rations expérimentales est grand.

La méthode classique des différences représente l'application de la méthode des équations dans le cas où l'on a :

$$
\mathrm{F}_{1}=1, \mathrm{C}_{1}=0, \mathrm{~F}_{2}=\mathrm{F}, \mathrm{C}_{2}=\mathrm{C}
$$

Dans cette partie de l'étude, il est apparu logique de ne pas reprendre ces dernières valeurs.

\section{Méthode par régression}

Elle résulte de l'ajustement d'un ensemble de résultats de mesures par le modèle linéaire suivant issu de l'équation (1):

$$
\text { CUD.r }=(\text { CUD.c }- \text { CUD.f }) C+\text { CUD.f }
$$

Les valeurs de CUD.r obtenues pour $\mathrm{C}=0$ et $\mathrm{C}=1$ permettent de connaître les valeurs des CUD attribuables aux composants du fourrage et de l'aliment concentré respectivement.

Si C désigne la valeur moyenne de la proportion du composant apportée par le concentré, l'écart-type de l'estimation de la digestibilité du foin est :

$$
\sigma(\text { CUD.f })=\sigma(\text { CUD.r }) \quad \sqrt{1+\frac{1}{n}+\frac{\bar{C}^{2}}{\sum(\mathrm{Ci}-\overline{\mathrm{C}})^{2}}}
$$

et celui de l'aliment concentré :

$$
\sigma(\text { CUD.c })=\sigma(\text { CUD.r }) \quad \sqrt{1+\frac{1}{n}+\frac{(1-\overline{\mathrm{C}})^{2}}{\sum(\mathrm{Ci}-\overline{\mathrm{C}})^{2}}}
$$


Cette approche présente, a priori, sur les autres l'intérêt de permettre de réaliser une analyse des variations résiduelles pour déterminer dans quelle mesure l'ajustement linéaire est le meilleur ou bien pour tenir compte de causes de variations dues à une interaction digestive (non linéarité) ou au niveau alimentaire (NA). S'ils ont une influence significative, ces différents facteurs peuvent alors être pris en compte grâce à l'emploi d'un modèle d’ajustement plus adapté, par exemple du type :

$$
\text { CUD.r }=a_{1} C+a_{2} C^{2}+a_{3} N A+a_{4}(N A)^{2}+a_{5}(N A)(C)+a_{6}
$$

\section{TABleAu 4}

Coefficients de digestibilité des différents principes de l'aliment et incertitudes correspondantes.

Cas de la méthode classique des différences (1).

Digestibility coefficients and related incertitudes of the different nutrients for concentrates.

Case of the classical difference method (1).

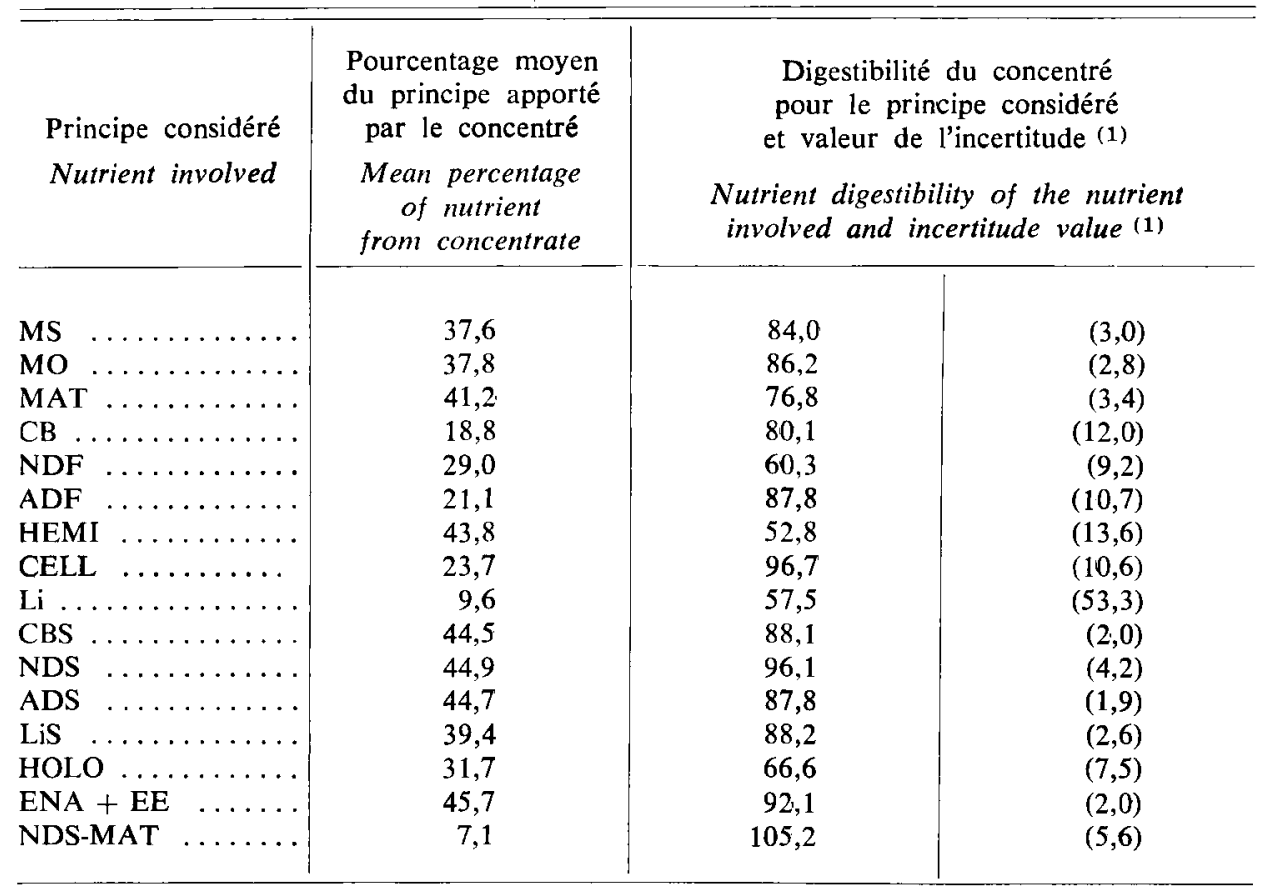

(1) $\mathrm{n}=18$ mesures -18 measures.

Incertitude traduite par le demi-intervalle de confiance - Incertitude expressed as the half interval of confidence. 


\section{Résultats}

\section{A. La méthode des différences}

Dans la première expérience, les bilans digestifs concernant le foin seul ont été effectués sur six chèvres au cours de deux périodes consécutives de cinq jours. Pour la seconde expérience, le nombre d'animaux est également de six, mais il n'y a eu qu'une période de mesure de bilans pour le foin. Pour chaque type daliment concentré considéré, 18 résultats individuels de digestibilité de la ration mixte ont été obtenus dans la première expérience et 12 dans la seconde. Le tableau 4 rapporte les résultats du calcul des CUD de différents principes de l'aliment concentré $\mathrm{R}$ obtenus par la méthode des différences à foin fixe. Dans le cas de la méthode classique, il est possible d’obtenir une valeur de l'incertitude qui s'attache à chaque coefficient de digestibilité par le calcul du demi-intervalle de confiance $: t_{1} \times \sigma($ CUD.c $) / \sqrt{n}$ à partir de $n=18$ résultats obtenus pour les rations mixtes (tabl. 4).

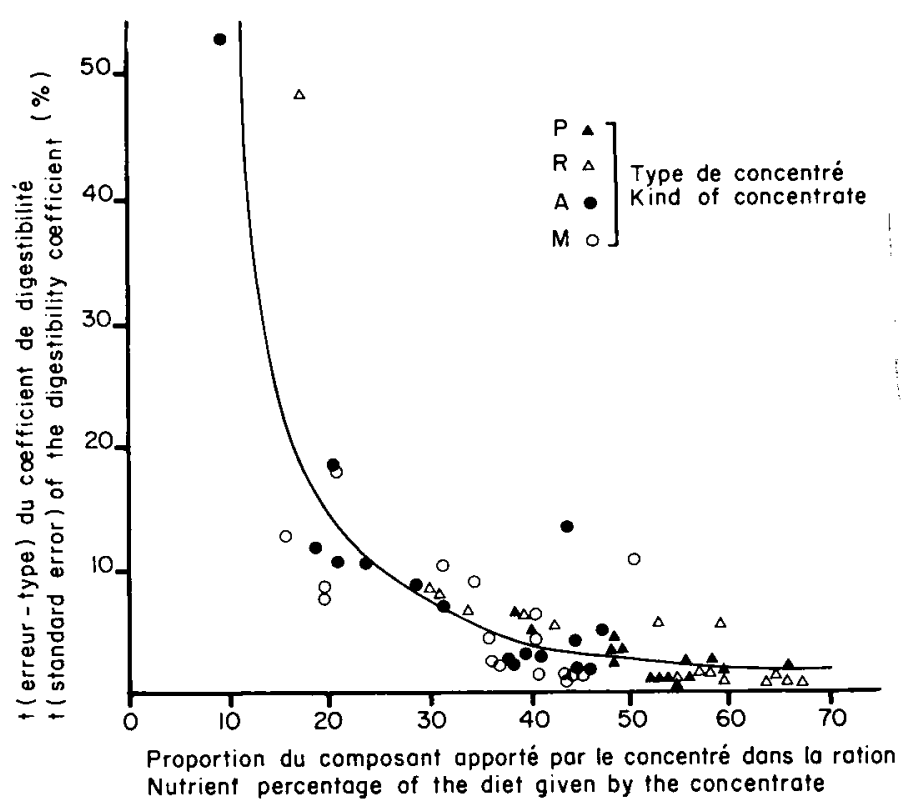

FIG. 2

Variations de la valeur du demi-intervalle de confiance du coefficient de digestibilité d'un composant d'un aliment concentré

en fonction de la proportion moyenne de ce composant apportée par le concentré.

Cas de la méthode classique des différences.

Variations in the half interval confidence value of the digestibility coefficient of a concentrate nutrient related to the mean percentage of this nutrient supplied by the concentrate.

(Classical difference method). 
La valeur de cette incertitude croît très rapidement lorsque la proportion moyenne du composant considéré apportée par l'aliment concentré est inférieure à 20-25 p. 100 (fig. 2). La pondération de chaque valeur obtenue par l'inverse de la proportion du composant ne modifie en moyenne statistiquement pas, avec les données considérées, la valeur des coefficients de digestibilité. En outre, pour les 64 valeurs calculées dans le cas où la digestibilité du fourrage est supposée être fixe (ff), la relation entre les deux séries pondérées (CUD. $\mathrm{ff}_{\mathrm{p}}$ ) ou non $\left(\right.$ CUD.ff $\mathrm{up}_{\mathrm{p}}$ ) est :

$$
\text { CUD.ff }_{\mathrm{np}}=1,07 \text { CUD.ff }_{\mathrm{p}}-5,39\left(\mathrm{r}=0,996, \mathrm{~S}_{\mathrm{sy}}=2,94, \mathrm{n}=64\right)
$$

La pondération modifie par contre fortement les incertitudes en resserrant l'éventail des valeurs (fig. 3). La plupart des données pondérées sont en effet associées à une incertitude située entre les valeurs de 3,5 et 7 p. 100 .

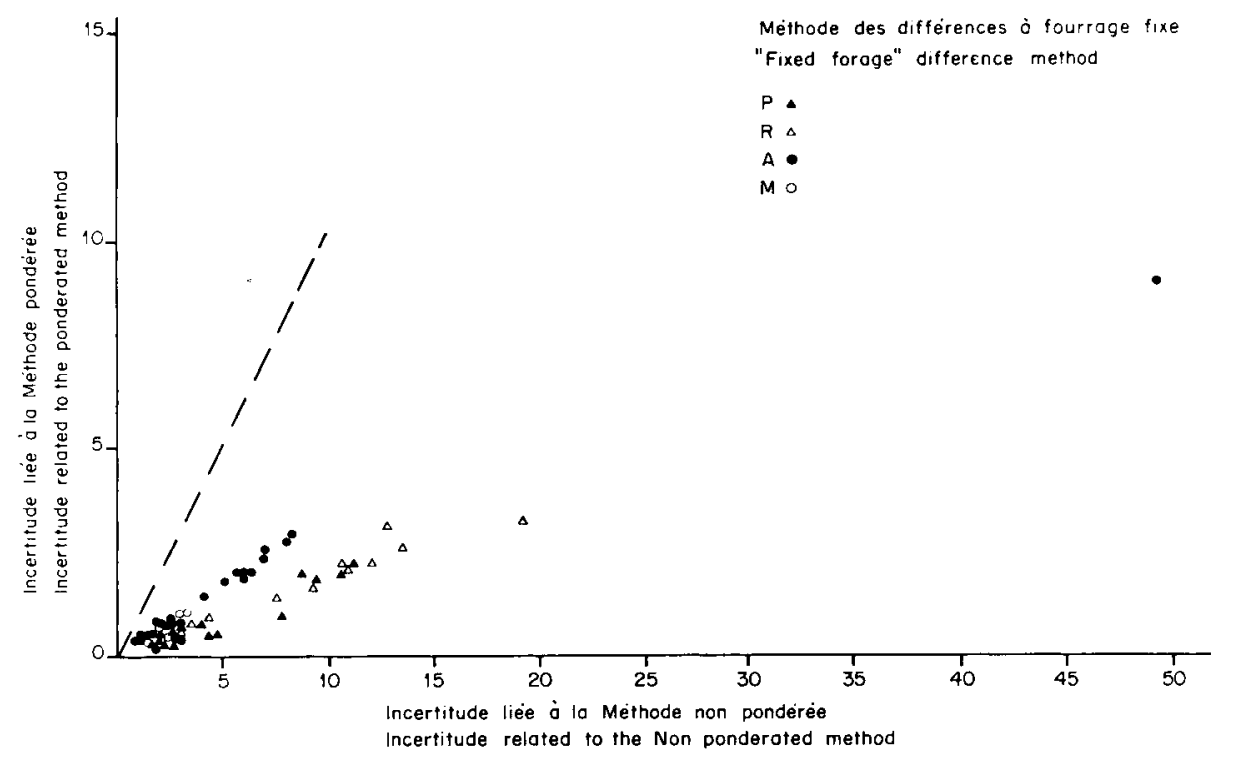

Fig. 3

Influence de la pondération sur la valeur de l'incertitude attachée à la digestibilité d'un aliment concentré.

Influence of ponderation on the incertitude value related to digestibility of a concentrate.

Lorsque les données enregistrées individuellement pour le foin sont prises en compte, l'expérience 1 correspond à 216 valeurs du coefficient de digestibilité pour chaque constituant; ce nombre est de 72 pour l'expérience 2 . La méthode de calcul à fourrage variable (fv), ne modifie statistiquement pas la valeur obtenue pour le 
coefficient de digestibilité (CUD.fv) en comparaison de la méthode à fourrage fixe (CUD.ff) :

$$
\begin{aligned}
& \text { CUD.f } \mathrm{v}_{\mathrm{np}}=1,00 \text { CUD.ff }_{\mathrm{np}}+0,18\left(\mathrm{R}=0,999, \mathrm{~S}_{\mathrm{xy}}=1,44, \mathrm{n}=64\right) \\
& (0,09) \quad(0,01)
\end{aligned}
$$

Par contre, l'incertitude qui s'attache à chaque valeur est fortement réduite (fig. 4). Comme pour la méthode à fourrage fixe, la prise en compte de la pondération inverse de la teneur en concentré ne modifie pas statistiquement la valeur des coefficients de digestibilité :

$$
\text { CUD.fv }_{\mathrm{p}}=\underset{(0,01)}{0,92 \text { CUD.fv }_{\mathrm{np}}}+\underset{(0,09)}{5,61}\left(\mathrm{R}=0,996, \mathrm{~S}_{\mathrm{xy}}=2,80, \mathrm{n}=64\right)
$$

Par contre, comme précédemment, la pondération réduit la valeur de l'incertitude qui est alors inférieure à la valeur de 2 pour 97 p. 100 des cas observés dans cette étude.

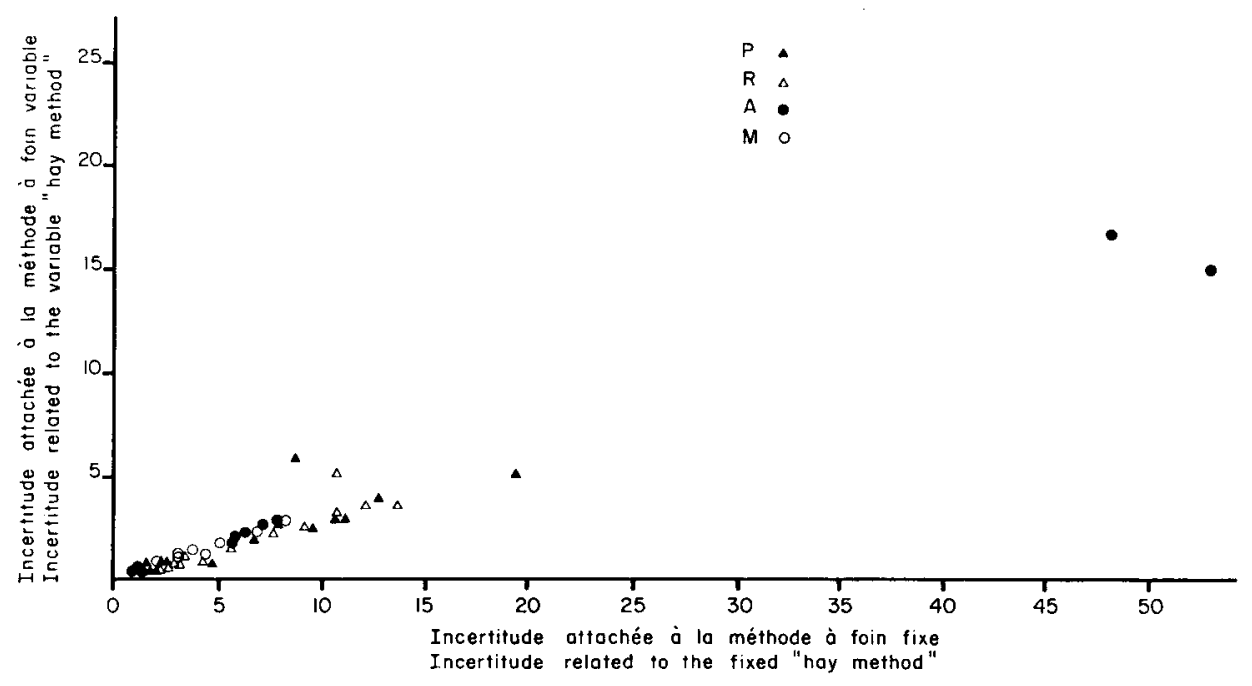

FIG. 4

Influence de la prise en compte des valeurs individuelles pour le foin sur la valeur de l'incertitude attachée à la digestibilité d'un aliment concentré. Méthode des différences non pondérées.

Influence of the use of individual hay values on the incertitude value related to the digestibility of a concentrate.

Non ponderated difference method.

Les histogrammes des valeurs obtenues pour les CUD de chaque composant ont été étudiés; avec la méthode des différences non pondérées à foin variable, ils suivent une loi normale dans 39 p. 100 des cas considérés (ex. fig. 5 : cas de la cellulose brute pour l'aliment M). Par contre, le rejet de la normalité de la distribution peut révéler l'existence d'une relation non linéaire entre C et CUD.c, c'est-à-dire un phéno- 
mène d'interaction digestive (fig. 6 : cas de la cellulose brute pour l'aliment A). Cependant, dans certains cas où la normalité est rejetée, cas de la matière organique pour l'aliment $\mathbf{R}$, un examen des données qui induisent des «queues de distribution» montre que ce sont celles qui correspondent à des bilans digestifs où la part de la matière organique apportée par le concentré est inférieure à $15 \mathrm{p}$. 100 . Si on fait, pour cet exemple, abstraction de ces 24 observations, la répartition devient normale (fig. 7).

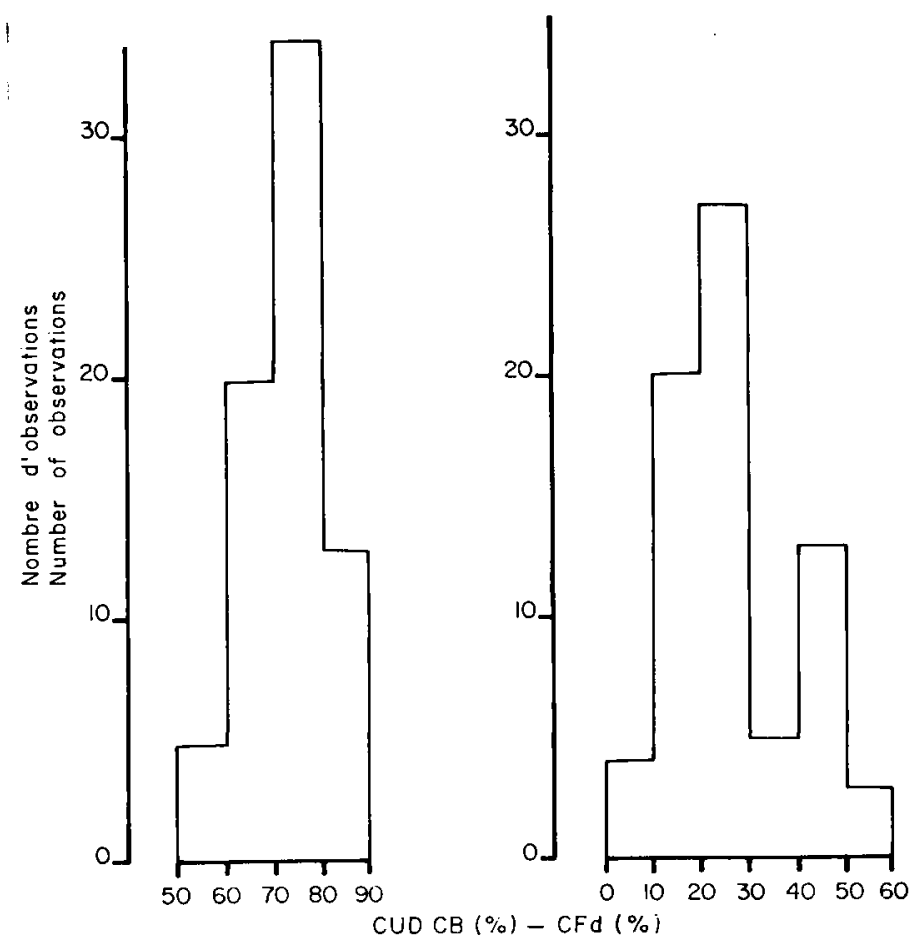

Fig. 5

Histogramme de la répartition du CUD de la cellulose brute (régime $M$ ). Histogram of crude fibre digestibility (diet $M)$.

FIG. 6

Histogramme de la répartition du CUD de la cellulose brute (régime A).

Histogram of crude fibre digestibility; calculated values (diet $A$ ).

\section{B. Résolution des systèmes d'équations}

Toutes les combinaisons possibles ont été, a priori, envisagées (une chèvre avec elle-même ou deux chèvres différentes). Cependant, lorsque les deux pourcentages d'aliment concentré (et donc de foin) pour un constituant donné sont très proches (moins 


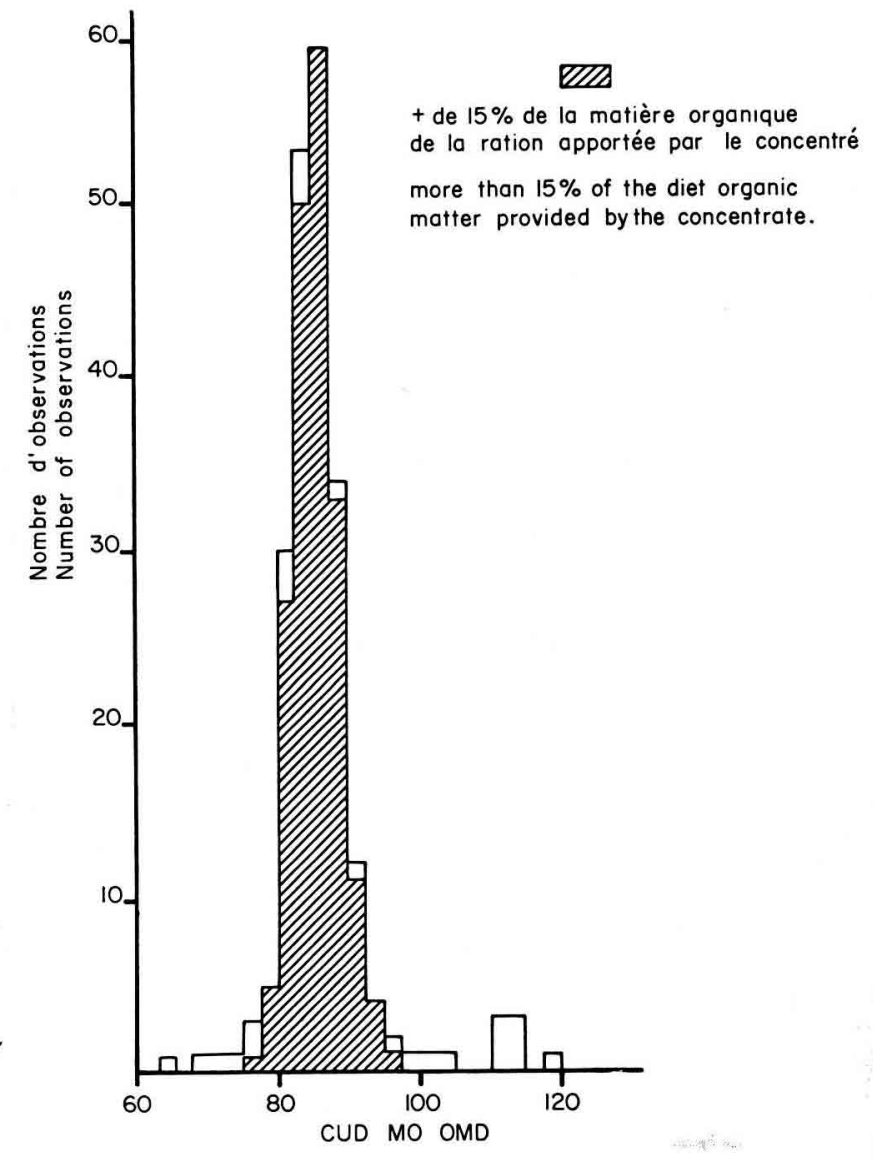

FIG. 7

Histogramme de répartition du coefficient de la matière organique (régime $R$ ).

Histogram of organic matter digestibility (diet $R$ ).

de 1 p. 100 d'écart entre les deux), les résultats obtenus peuvent être aberrants. De ce fait, seules ont été prises en considération les valeurs obtenues avec des différences de pourcentage entre les deux observations supérieures à 1 p. 100. Ces calculs, faits pour chaque couple foin-concentré, aboutissent à un ensemble de coefficients de digestibilité, a priori très dispersés (fig. 8) qu'il convient de trier en fonction d'un objectif de maximisation de la précision du résultat. Lorsque $\left|\mathrm{C}_{2}-\mathrm{C}_{1}\right|$ croît, le nombre de données disponibles comme base du calcul s'amenuise. En conséquence, pour déterminer le meilleur ensemble des résultats à retenir pour calculer CUD.c, les données ont été tronquées par étape en augmentant à chaque fois de 2,5 p. 100 la valeur du seuil minimal de $\left|\mathrm{C}_{2}-\mathrm{C}_{1}\right|$. 


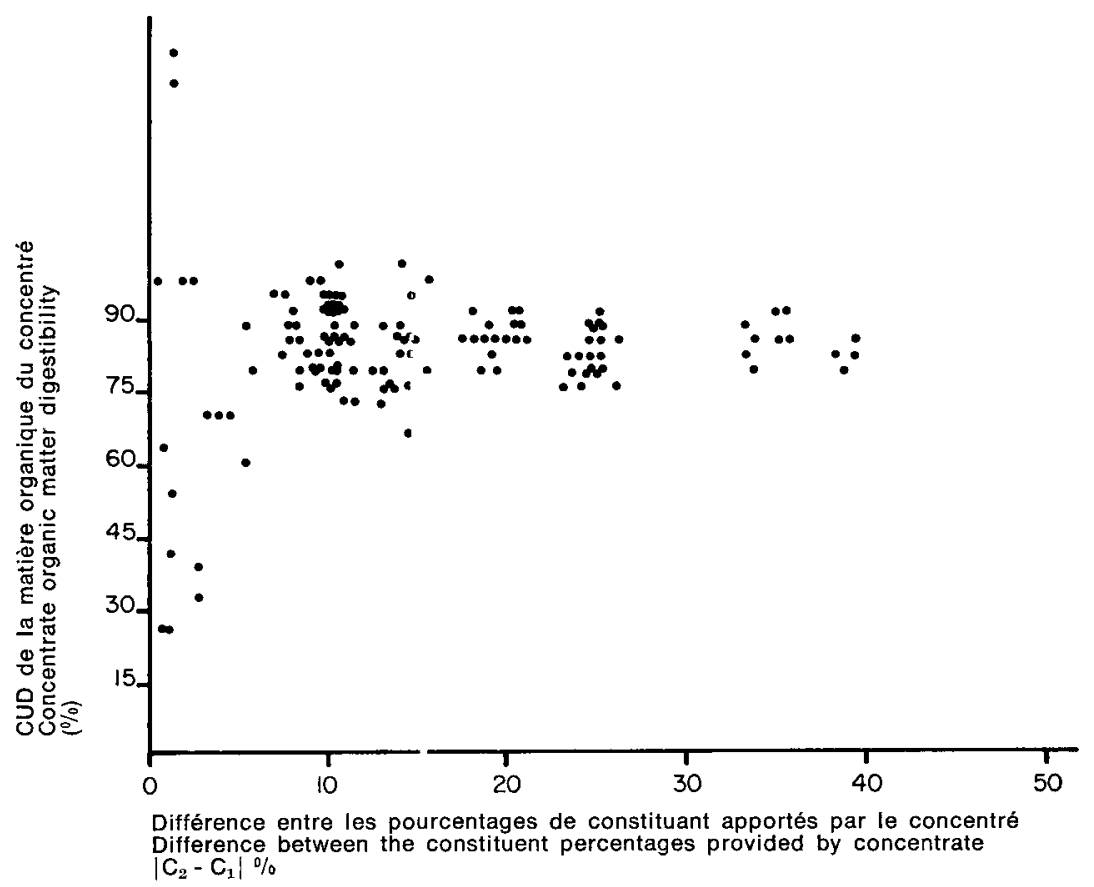

FIG. 8

Relation entre le CUD de la matière organique du concentré obtenue par la méthode des équations et la différence entre les pourcentages de constituant apporté par le concentré (régime R).

Relationships between the concentrate organic matter digestibility obtained by the equation method and the difference beiween constituent percentages provided by concentrate (diet $R$ ).

A chaque étape et pour chaque composant, l'incertitude attachée à CUD.c, c'est-àdire le produit $t_{n} \times \sigma$ (CUD.c) $/ \sqrt{n}$, a été calculée pour les valeurs retenues. Pour tous les composants, cette incertitude passe par une valeur minimale (fig. 9). Le repérage du minimum permet de déterminer les quatre paramètres suivants :

- la valeur du coefficient CUD.c ;

- l'incertitude attachée à cette valeur de CUD.c;

- le niveau de troncage, c'est-à-dire la valeur de $\left|\mathrm{C}_{2}-\mathrm{C}_{1}\right|$ associée au minimum de l'incertitude ;

- la proportion des données de départ retenues.

En pratique, l'incertitude passe souvent par un «plateau de valeurs minimales » en fonction des variations de $\left|C_{2}-C_{1}\right|$ (fig. 9). Ce phénomène rend délicate la détermination exacte du minimum; de ce fait, pour comparer les différentes méthodes, les valeurs de seuil retenues correspondent à la moyenne des trois valeurs consécutives les plus basses de l'incertitude. 


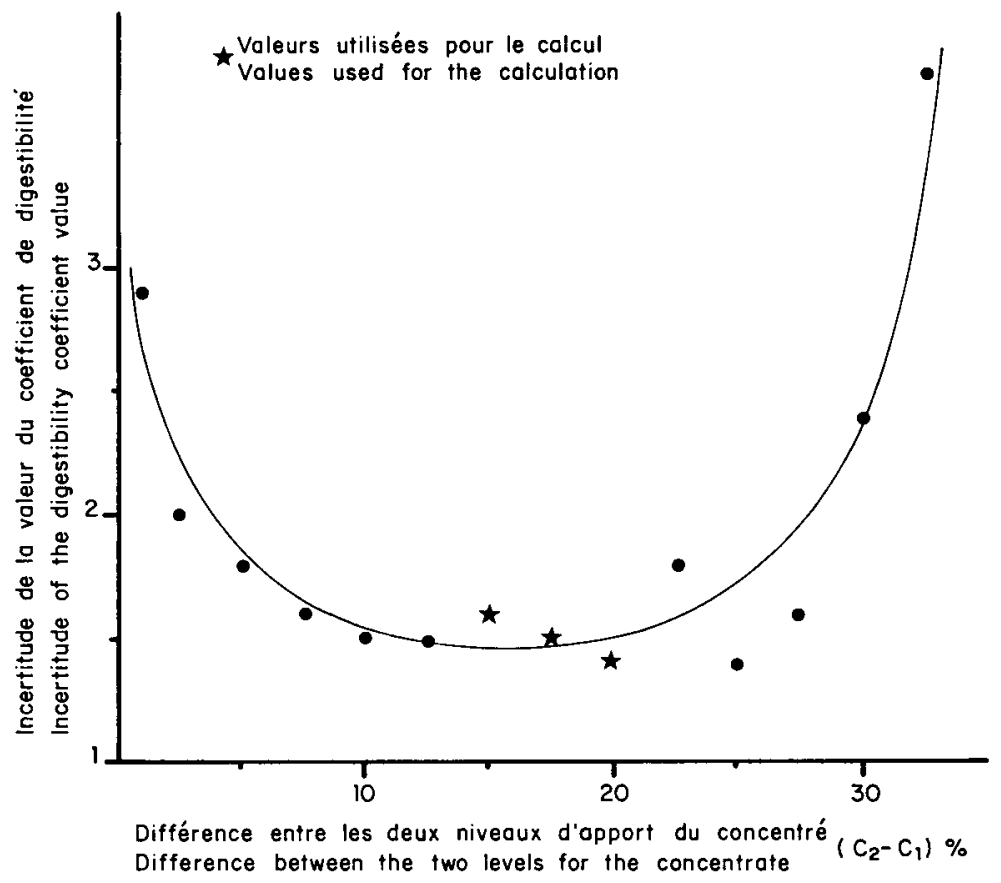

FIG. 9

Evolution de l'incertitude du coefficient de digestibilité du résidu NDF d'un aliment concentré dans la méthode des équations (1).

Incertitude of the cell-wall digestibility of a concentrate by the equation method (1).

(1) Valeurs utilisées pour le calcul $\star$-Values used for the calculation.

Cette démarche a été systématiquement appliquée pour chaque constituant des foins et aliments concentrés des deux expériences, c'est-à-dire à 120 ensembles de 144 ou 60 données chacun. Le niveau du troncage a été en moyenne de 10,3 p. 100 ( $\mathrm{s}=4,2$ p. 100$)$ et de 12,1 p. $100(\mathrm{~s}=6,0$ p. 100) respectivement pour l'aliment concentré et le foin. Ce niveau a été en moyenne plus bas pour la seconde expérimentation que pour la première. Les proportions de données retenues ont été de 46,4 p. 100 ( $s=17,1$ p. 100$)$ et de 41,2 p. $100(s=16,3$ p. 100) respectivement pour l'aliment concentré et le foin. Pour chaque principe et expérience considérés, les niveaux de troncage et les proportions de données retenues pour le calcul sont proches entre le foin et l'aliment concentré ; en effet, pour 60 données, les corrélations ont été de 0,81 pour le niveau de troncage et 0,68 pour la proportion de données retenues. Par contre, pour un type donné d'aliment, foin ou concentré, la proportion de données retenues diminue lorsque le niveau du troncage s'élève. En effet, sur 60 valeurs, les corrélations calculées entre ces deux caractéristiques sont de $-0,62$ et $-0,75$ respectivement pour l'aliment concentré et le foin. 


\section{Méthode par régression}

Les calculs par régression ont été effectués, dans un premier temps, avec le modèle linéaire simple en fonction de la proportion $C$ de l'élément considéré apporté par le concentré. Ces ajustements ne sont pas tous statistiquement significatifs, notamment lorsque le pourcentage moyen du principe apporté par le concentré est inférieur à 20 p. 100 ou lorsque les CUD de l'élément considéré du foin et de l'aliment concentré sont proches. Le minimum d'écart entre ces deux valeurs de CUD est de l'ordre de 5 points avec les résultats obtenus. Dans la plupart des cas où la proportion $\mathrm{C}$ de l'élément représenté par le concentré n'a pas d'influence significative, les autres caractéristiques prises en compte dans le modèle ne modifient pas la situation. Cependant, quelques exceptions ont été observées : ainsi pour l'aliment concentré $A$, la prise en compte du niveau alimentaire (NA) permet d'objenir un ajustement statistiquement significatif de la digestibilité de la cellulose brute :

$$
\begin{aligned}
\mathrm{CUD}_{\mathrm{CB}}= & 45,94-20,73 \mathrm{C} \\
& \left(\mathrm{n}=12, \mathrm{R}=0,34, \mathrm{~S}_{\mathrm{xy}}=4,00\right) \\
\mathrm{CUD}_{\mathrm{CB}}= & 45,03-78,03 \mathrm{C}+23,0 \mathrm{C} \times \mathrm{NA} \\
& \left(\mathrm{n}=12, \mathrm{R}=0,78, \mathrm{~S}_{\mathrm{xy}}=2,81\right)
\end{aligned}
$$

Pour ces deux modèles, l'estimation de la digestibilité de la cellulose brute du foin est comparable $(45,9$ vs 45,0$)$ comme pour l'aliment concentré $(25,2$ vs 25,3$)$. Lorsque l'ajustement linéaire est significatif, la proportion du principe dans l'aliment concentré ne constitue pas toujours la variable la plus corrélée avec le coefficient de digestibilité ; de ce fait, dans les calculs par régression multiple progressive, la première variable explicative entrante a été, par exemple, le niveau d'alimentation, le carré de la proportion de concentré... Cependant, lorsque la proportion $\mathrm{C}$ du composant du concentré est imposée comme première variable entrante de la régression, les autres variables évoquées n'ont plus d'influence significative. Un seul résultat a fait exception : il s'agit de la digestibilité de la fraction «hémicellulosique ( NDF-ADF) dans le cas du régime riche $(R)$. Dans ce cas, le niveau alimentaire a présenté une influence spécifique significative :

$$
\begin{aligned}
\operatorname{CUD}_{(\mathrm{NDF}-A \mathrm{AF})}= & 46,30+39,38 \mathrm{C} \\
& \left(\mathrm{n}=18, \mathrm{R}=0,92, \mathrm{~S}_{\mathrm{xy}}=2,21\right) \\
\operatorname{CUD}_{(\mathrm{NDF}-A \mathrm{DF})}= & 47,40+63,05 \mathrm{C}-6,17 \mathrm{NA} \\
& \left(\mathrm{n}=18, \mathrm{R}=0,94, \mathrm{~S}_{\mathrm{xy}}=1,97\right)
\end{aligned}
$$

\section{Comparaison simultanée des différentes méthodes}

Le tableau 5 rapporte les valeurs des coefficients de corrélation entre les valeurs des CUD des différents composants des aliments concentrés issues des six méthodes d'estimation. Les valeurs des coefficients de digestibilité sont hautement corrélées entre les méthodes procédant par différence; en outre dans ces cas, les droites de régression ne s'écartent statistiquement pas de la «première bissectrice». Les résultats sont également très comparables entre la méthode des équations et celle des régressions. Par contre, les relations sont moins étroites entre ces deux dernières méthodes et le groupe de celles qui procèdent par différence. Pour le foin, il y a également une relation 
étroite entre les valeurs des coefficients de digestibilité calculées par les équations et par régression (tabl. 6). Par contre, ces valeurs ne sont que moyennement corrélées aux valeurs mesurées lorsque le foin était distribué seul (tabl. 6, fig. 10).

\section{TABLEAU 5}

Corrélations entre les coefficients de digestibilité obtenus pour l'aliment concentré par les différentes méthodes (1).

Correlations between the concentrate digestibility values obtained by different ways of calculation (1).

Méthode des différences classique ...... Classical difference method

Méthode des différences à foin variable .. «Variable hay» difference method

Méthode des différences pondérée ....... «Ponderated hay» difference method

Méthode des différences pondérée à foin variable ..................... «Ponderated variable hay» difference method

Méthode des équations

Equation method

Méthode des régressions .............

Regression method
1,000

$0,998^{* *} 1,000$

$0,997 * 0,993 * 1,000$

$0,997 * * 0,996 * * 0,999 * * 1,000$

$0,858 * 0,850 * * 0,889 * * 0,884 * * 1,000$

$0,886 * 0,876 * 0,916 * 0,909 * * 0,979 * * 1,000$

(1) 62 degrés de liberté - 62 degrees of freedom.

Seuil de signification : Level of significance : $* * \mathrm{p}<0,01: 0,325$.

\section{TABLEAU 6}

Corrélations entre les coefficients de digestibilités obtenus pour le foin par les différentes méthodes (1).

Correlations between the hay digestibility values obtained by different ways of calculation (1).

Digestibilité mesurée in vivo $\ldots \ldots \ldots \ldots \ldots \ldots \ldots \ldots \ldots \ldots$

In vivo digestibility

Digestibilité issue de la méthode des équations $\ldots \ldots \ldots \ldots \ldots, 0,795^{* *} \quad 1,00$

Digestibility obtained by the equation method

Digestibilité issue de la méthode des régression

$0,794^{* *} \quad 0,996^{* *} \quad 1,00$

Digestibility obtained by the regression method

(1) 62 degrés de liberté - 62 degrees of freedom.

Seuil de signification : Level of significance : *** $p<0,01: 0,325$. 


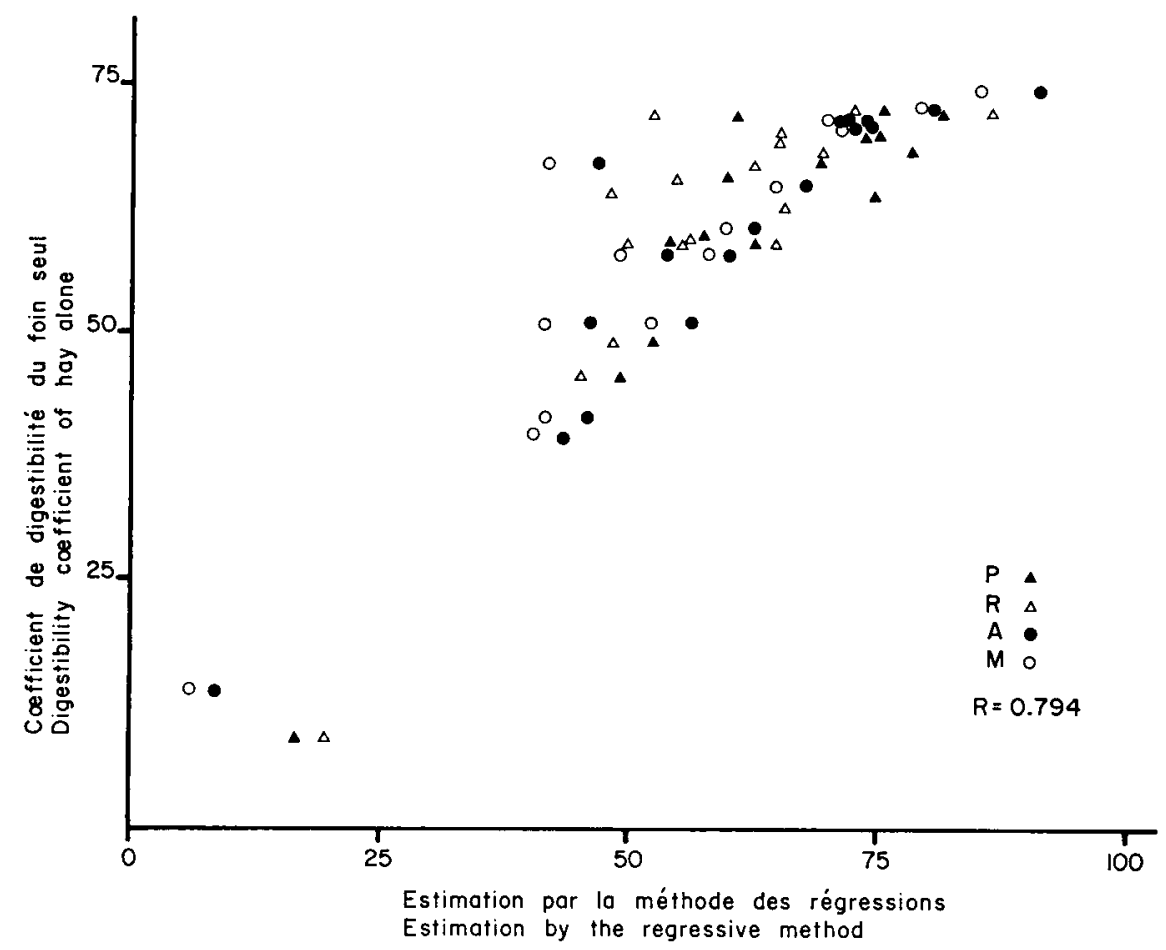

Fig. 10

Comparaison de deux méthodes d'estimation du coefficient de digestibilité des différents constituants du foin.

Comparison of two methods for nutrient digestibility calculation of hay.

Dans 7 cas sur 64, le CUD d'un principe du foin obtenu par régression diffère significativement (seuil de 5 p. 100) de celui du même principe lorsque le foin est distribué seul. La figure 10 indique que pour les composants les plus digestes du foin mesuré seul, la méthode par régression fournit des estimations de digestibilité très variables. Le tableau 7 rapporte les corrélations observées entre les incertitudes attachées aux différentes valeurs des CUD. Les variations de l'incertitude sont très corrélées entre les méthodes des équations et par régression. Il en va de même pour les deux méthodes des différences non pondérées d'une part, et les deux méthodes des différences pondérées d'autre part. Les incertitudes obtenues avec les méthodes des différences non pondérées sont positivement mais moyennement corrélées à celles obtenues avec les quatre autres méthodes (différences pondérées avec ou sans fourrage variable, équations, régression). Les relations sont par contre inverses entre les incertitudes liées aux méthodes dites des différences pondérées, en particulier la méthode des différences pondérées à foin fixe et les méthodes des équations ou par régression.

Pour pouvoir procéder à une analyse plus détaillée des relations observées entre les valeurs des coefficients de digestibilité, des incertitudes et d'autres caractéristiques 
mesurées au cours des expérimentations, l'ensemble des données a été traité par analyse en composantes principales. Le tableau 8 indique l'ensemble des caractéristiques considérées, leurs sigles et leurs moyennes et écart type.

\section{TABLEAU 7}

Tableau des corrélations entre les incertitudes obtenues pour les différentes méthodes d'estimation des coefficients de digestibilité des aliments concentrés (1).

Table of correlations about incertitudes obtained by the different methods to estimate the digestibility value of concentrates (1).

Différence

Différence foin variable

«Variable hay $»$ difference

Différence pondérée

Ponderated difference

Différence pondérée foin variable ......

Ponderated «variable hay » difference

Equation

Equation

Régression

Regression
1

$0,91 * * \quad 1$

$0,97 * * \quad 0,71 * * \quad 1$

$0,88^{* *} \quad 0,97 * * \quad 0,91 * * \quad 1$

$0,43 * * \quad 0,51 * * \quad 0,44 * * 0,52 * * 1$

$0,38 * * 0,45 * * \quad 0,39 * * \quad 0,45 * * \quad 0,95 * * 1$

(1) Corrélations calculées à partir de 64 observations - Correlations obtained from 64 observations. Seuil de signification - Level of significance :

** $\mathrm{p}<0,01: 0,325$.

$* \mathrm{p}<0,05: 0,250$.

La première composante principale $(41,5$ p. 100 de variance expliquée) traduit des différences associées à la valeur des coefficients de digestibilité. La corrélation linéaire est plus élevée pour les composants ayant le meilleur CUD ; en outre, l'écart type résiduel associé à la régression linéaire ainsi que l'ensemble des incertitudes, mises à part celles des méthodes des différences pondérées, sont plus faibles (fig. 11). En raison de la symétrie de l'effet traduit par une composante principale, des caractéristiques inverses sont associées aux composantes les moins digestes.

La caractéristique explicative la plus liée à cette première composante, est la proportion $\mathrm{C}$ du constituant considérée apportée par l'aliment concentré, cette proportion étant en moyenne plus faible dans le cas des composants pariétaux qui sont en teneur plus élevée dans les foins. 


\section{Tableau 8}

Sigles, moyenne et écart type des variables considérées dans l'analyse des composantes principales.

Symbol, mean and standard error of variables used in the principal component analysis.

Variable
Variable

p. 100 de concentré dans la ration .... p. 100 of concentrate in the diet

Ecart type du (----) Standard deviation of (----)

Digestibilité du constituant dans la ration Digestibility of component in the diet Ecart type du (..--) ............... Standard deviation of (--.-)

p. 100 moyen du constituant apporté par le concentré ................... Mean percentage of component provided by concentrate

Digestibilité du foin seul ............

Digestibility of hay alone

Ecart type du (---) ..............

Standard deviation of (-----)

Méthode des différences

Difference method

1) Classique Usual

Digestibilité du concentré Digestibility of concentrate

Ecart type du (---) ................ Standard deviation of (----)

2) Pondérée à foin fixe Ponderated with fixed hay

Digestibilité du concentré Digestibility of concentrate

Ecart type du (----) Standard deviation of (----)

3) Non pondérée à foin variable Non ponderated with variable hay

Digestibilité du concentré Digestibility of concentrate

\begin{tabular}{|c|c|c|}
\hline $\begin{array}{l}\text { Sigle } \\
\text { Symbol }\end{array}$ & $\begin{array}{l}\text { Moyenne } \\
\text { Mean }\end{array}$ & $\begin{array}{c}\text { Ecart type } \\
\text { Standard error }\end{array}$ \\
\hline $\begin{array}{c}\text { p. } 100 \\
\text { CONCRAT }\end{array}$ & 44,6 & 13,1 \\
\hline $\begin{array}{l}\text { S p. } 100 \\
\text { CONRAT }\end{array}$ & 7,78 & 4,19 \\
\hline DIGCONRA & 67,8 & 14,1 \\
\hline SDIG RAT & 3,00 & 1,84 \\
\hline PCONMOY & 42,5 & 13,8 \\
\hline DIGF DIF & 62,7 & 9,68 \\
\hline SDIGFDIF & 1,35 & 0,72 \\
\hline DIGC DIF & 73,3 & 23,2 \\
\hline SDIGCDIF & 4,53 & 3,47 \\
\hline DIGC DIPO & 73,4 & 22,2 \\
\hline SDIGCDIP & 5,60 & 1,33 \\
\hline DIGCDPNV & 73,6 & 23,3 \\
\hline
\end{tabular}


Tableau 8 (suite).

\begin{tabular}{|c|c|c|c|}
\hline $\begin{array}{l}\text { Variable } \\
\text { Variable }\end{array}$ & $\begin{array}{c}\text { Sigle } \\
\text { Symbol }\end{array}$ & $\begin{array}{l}\text { Moyenne } \\
\text { Mean }\end{array}$ & $\begin{array}{l}\text { Ecart type } \\
\text { Standard error }\end{array}$ \\
\hline $\begin{array}{l}\text { Ecart type du (---) } \ldots \ldots \ldots \ldots \ldots \ldots \\
\text { Standard deviation of }(----)\end{array}$ & SDIGCPNV & 1,50 & 1,22 \\
\hline $\begin{array}{l}\text { 4) Pondérée à foin variable } \\
\text { Ponderated with variable hay }\end{array}$ & & & \\
\hline $\begin{array}{l}\text { Digestibilité du concentré } \ldots \ldots \ldots \ldots \ldots \\
\text { Digestibility of concentrate }\end{array}$ & DIGCDP V & 73,6 & 22,3 \\
\hline $\begin{array}{l}\text { Ecart type du (---) } \ldots \ldots \ldots \ldots \ldots \ldots \\
\text { Standard deviation of }(---)\end{array}$ & SDIGCDPV & 1,67 & 0,29 \\
\hline $\begin{array}{l}\text { Méthode des équations } \\
\text { Equation method }\end{array}$ & & & \\
\hline $\begin{array}{l}\text { Digestibilité du foin } \ldots \ldots \ldots \ldots \ldots \ldots \\
\text { Digestibility of hay }\end{array}$ & DIGF EQU & 62,0 & 12,4 \\
\hline $\begin{array}{l}\text { Ecart type du (---) } \ldots \ldots \ldots \ldots \ldots \cdots \\
\text { Standard deviation of (----) }\end{array}$ & SDIGFEQU & 2,92 & 2,31 \\
\hline $\begin{array}{l}\text { p. } 100 \text { de données retenues pour le foin } \\
\text { p. } 100 \text { retained observations for hay }\end{array}$ & RETENU & 41,2 & 16,3 \\
\hline $\begin{array}{l}\text { Niveau de troncage pour le foin } \ldots \\
\text { Truncation level for hay }\end{array}$ & NIVFTRON & 12,1 & 5,97 \\
\hline $\begin{array}{l}\text { Digestibilité du concentré } \ldots \ldots \ldots \ldots \\
\text { Digestibility of concentrate }\end{array}$ & DIGC EQU & 73,9 & 18,1 \\
\hline $\begin{array}{l}\text { Ecart type du (---) } \ldots \ldots \ldots \ldots \ldots \ldots \\
\text { Standard deviation of (--) }\end{array}$ & SDIGCEQU & 3,54 & 2,73 \\
\hline $\begin{array}{l}\text { p. } 100 \text { de données retenues pour le } \\
\text { concentré } \ldots \ldots \ldots \ldots \ldots \ldots \ldots \ldots \ldots \ldots \ldots \\
\text { p. } 100 \text { of retained observations for hay }\end{array}$ & RETENU & 46,4 & 17,1 \\
\hline $\begin{array}{l}\text { Niveau de troncage pour le concentré .. } \\
\text { Truncation ievel for concentrate }\end{array}$ & NIVCTRON & 10,3 & 4,17 \\
\hline $\begin{array}{l}\text { Méthode des régressions } \\
\text { Regression method }\end{array}$ & & & \\
\hline $\begin{array}{l}\text { Digestibilité du foin } \ldots \ldots \ldots \ldots \ldots \ldots \\
\text { Digestibility of hay }\end{array}$ & DIGF REG & 61,9 & 12,5 \\
\hline $\begin{array}{l}\text { Ecart type du }(---)(\ldots \ldots \ldots \ldots \ldots \ldots \\
\text { Standard deviation of }(-\ldots)\end{array}$ & SDIGFREG & 2,45 & 1,74 \\
\hline $\begin{array}{l}\text { Digestibilité du concentré } \ldots \ldots \ldots \ldots \ldots \\
\text { Digestibility of concentrate }\end{array}$ & DIGC REG & 73,9 & 18,1 \\
\hline $\begin{array}{l}\text { Ecart type du (---) } \ldots \ldots \ldots \ldots \ldots \ldots \\
\text { Standard deviation of (--.-) }\end{array}$ & SDIGCREG & 2,71 & 1,97 \\
\hline $\begin{array}{l}\text { Coefficient de corrélation } \ldots \ldots \ldots \ldots \ldots \\
\text { Correlation coefficient }\end{array}$ & CORREL & 0,60 & 0,24 \\
\hline $\begin{array}{l}\text { Ecart type du (---) } \ldots \ldots \ldots \ldots \ldots \ldots \\
\text { Standard deviation of }(---)\end{array}$ & S CORREL & 1,89 & 0,91 \\
\hline
\end{tabular}




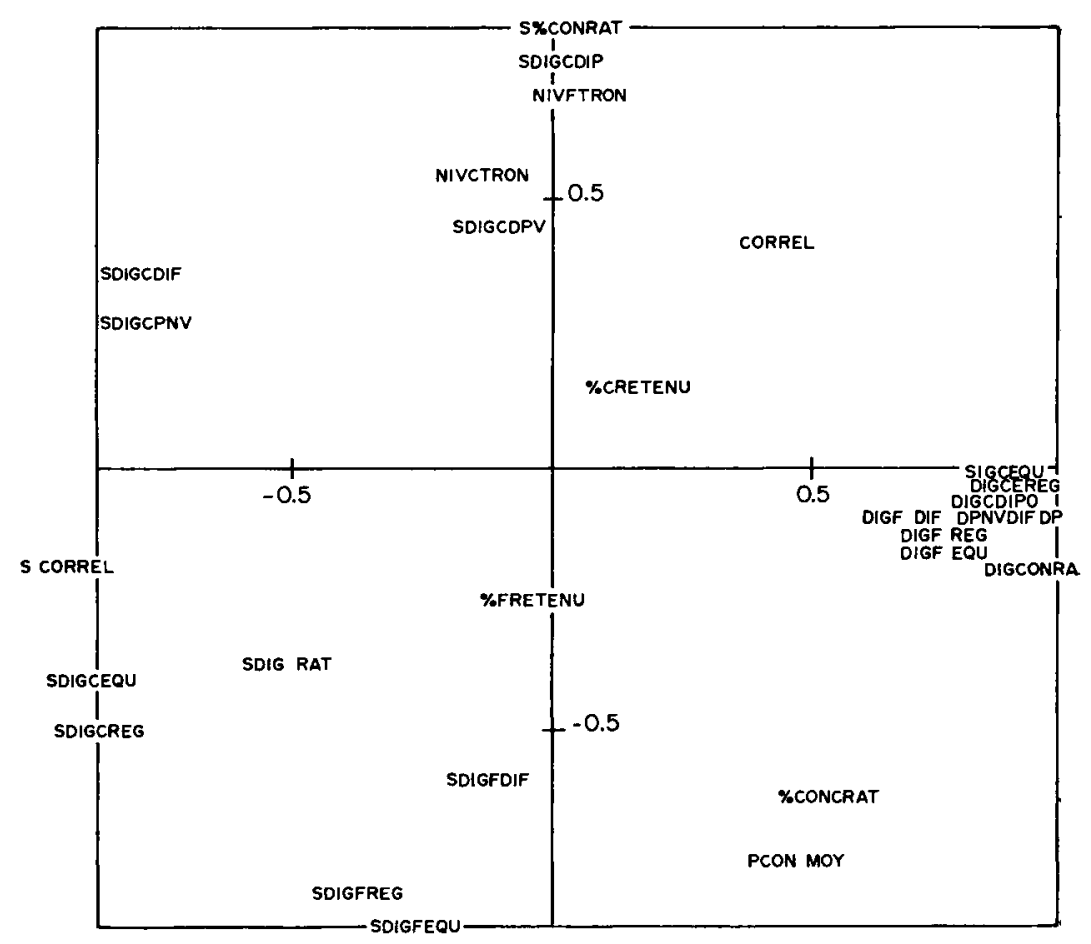

FIG. 11

Projection des variables

sur le plan déterminé par les deux premières composantes principales (ACP).

Projection of variables on the two first components plan $(A C P)$.

La seconde composante principale (21,9 p. 100 de variance expliquée) indique que les variations de la proportion de l'aliment concentré n'ont pas systématiquement eu les effets mis en évidence par la première composante. Dans les situations correspondant à une proportion plus élevée mais peu variable d'aliments concentrés (cf. p. 100 CONCRAT et $\mathbf{S}$ p. 100 CONRAT), l'incertitude des coefficients de digestibilité de l'aliment concentré est plus importante pour les méthodes des équations et par régression, mais plus réduite pour les méthodes des différences, pondérées en particulier. Dans ces situations, le niveau de troncage est en moyenne plus faible et l'incertitude sur le CUD du foin, par les méthodes des équations et par régression, est encore plus forte que celle obtenue pour les aliments concentrés (fig. 11). La projection des points observations sur cette composante montre qu'elle tend à discriminer les deux expérimentations considérées dans cette étude; en outre, au sein de chacune d'elles, les résultats relatifs aux deux types d'aliments concentrés se distinguent.

La troisième composante ( 12 p. 100 de variance expliquée) traduit les variations opposées entre les proportions de données retenues et le niveau de troncage dans le cas 
de la méthode des équations. Il y a plus de données retenues lorsque le coefficient de corrélation lié à la méthode des régressions est plus élevée. Dans ces situations, l'incertitude attachée aux méthodes pondérées est plus forte. D'autre part, lorsque plus de données ont été retenues, la méthode des équations et celle par régression tendent à aboutir, en comparaison des méthodes des différences, à des valeurs de digestibilité supérieures pour l'aliment concentré et inférieures pour le foin (fig. 12).

La quatrième composante $(6,4$ p. 100 de variance expliquée) traduit des écarts de CUD entre les méthodes des différences et les autres. Ces écarts sont associés aux variations de la proportion de concentré. Lorsque cette proportion augmente, la méthode des différences fournit des valeurs de digestibilité supérieures pour le foin et inférieures pour l'aliment concentré (fig. 12). Les projections des points observations sur cet axe indiquent que ce sont les constituants pariétaux qui sont les plus discriminés par cette composante.

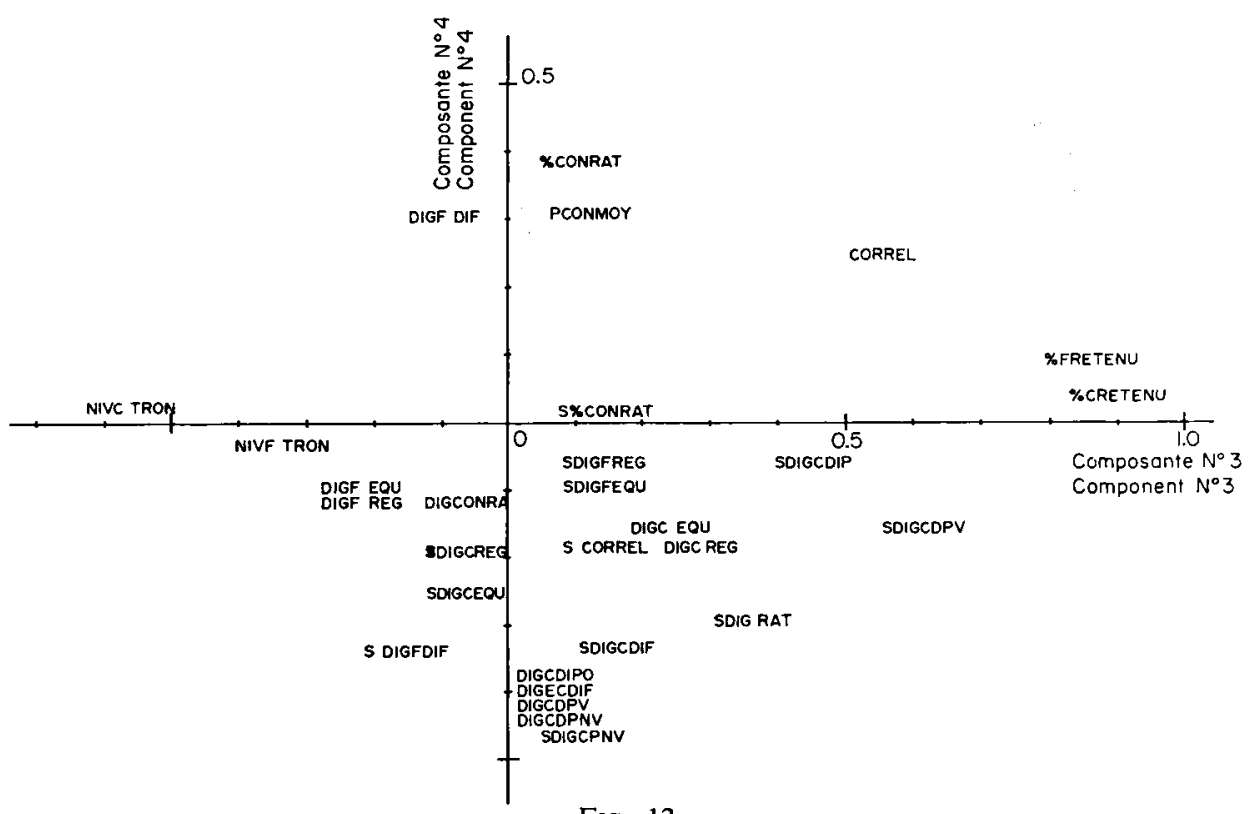

FiG. 12

Projection des variables sur le plan des composantes 3 et 4 (ACP).

Projection of variables on the plan determined by components 3 and 4 (ACP).

Bien que traduisant une plus faible explication de la variance des données, cette quatrième composante prend en compte des variations importantes de digestibilité liées à la méthode de calcul. Ainsi, dans le cas de l'al:ment $\mathrm{R}$ 《riche », l'application de la régression aboutit à accroître le coefficient de digestibilité de la fraction ADF du foin de 3,8 points et de réduire celui de l'aliment concentré de 18,9 points. 


\section{Discussion}

Les données expérimentales utilisées dans ce travail ont concerné des plages de variations importantes de proportion d'aliments concentrés, de 13,5 p. 100 à 67,6 p. 100, et de niveaux alimentaires, de 1,03 à 3,36. Par contre, seulement quatre types de régimes alimentaires ont été testés; ce fait limite a priori la portée des résultats. Il convient cependant de rappeler que le mode de détermination de la composition des aliments concentrés a permis de se placer dans des situations plutôt extrêmes par rapport aux plages de variation de composition et de digestibilité observées en pratique (SAUVANT \& Giger, 1980).

\section{A. Les méthodes des différences}

Avec les méthodes des différences non pondérales, l'incertitude s'accroît fortement, comme l'ont déjà montré Charlet-Lery \& Leroy (1955) et Frederiksen (1973), lorsque la proportion du principe apporté par l'aliment concentré est inférieure à 2025 p. 100. Il serait indispensable, d'une part, d'intégrer dans la conception des mesures de digestibilité, ces variations de la fiabilité des mesures et, d'autre part, d'indiquer systématiquement l'incertitude attachée aux différents coefficients de digestibilité publiés, ou au moins la proportion de la matière sèche de la ration apportée par l'aliment concentré. Si MSC désigne cette proportion et si $\mathrm{c}$ et $\mathrm{f}$ représentent la teneur d'un composant dans la matière sèche du concentré et du fourrage et si $\ell$ représente la limite minimale de la proportion du composant apporté par l'aliment concentré, ces différents paramètres sont associés dans la relation :

$$
\frac{\mathrm{c.MSC}+\mathrm{f}(1-\mathrm{MSC})}{\mathrm{c.MSC}} \geqslant \ell
$$

Cette relation peut être utilisée de plusieurs façons; ainsi la figure 13 rapporte des abaques qui permettent de déterminer la valeur minimale de MSC en fonction de $f$ pour un seuil limite de 25 p. 100 au moins du composant considéré apporté par l'aliment concentré. Par exemple, pour mesurer le coefficient de digestibilité de la cellulose brute d'une orge en contenant $50 \mathrm{~g} / \mathrm{kg}$ MS avec un foin de luzerne en contenant $350 \mathrm{~g} / \mathrm{kg} \mathrm{MS}$, les abaques indiquent que l'orge devra représenter au moins $70 \mathrm{p} .100$ de la matière sèche de la ration. Les abaques indiquent également que lorsqu'on limite le niveau d'apport du concentré, à 60 p. 100 de la MS par exemple, il n'est pas possible d'obtenir une valeur fiable du CUD de la cellulose brute d'un aliment concentré en contenant moins de $68 \mathrm{~g} / \mathrm{kg}$ MS si la mesure est réalisée avec un foin contenant $300 \mathrm{~g}$ de cellulose brute/ $\mathrm{kg}$ MS.

La valeur énergétique d'un aliment concentré peut être prédite avec précision à partir des teneurs en éléments digestibles (SCHIEMAN et al., 1971) :

$$
\mathrm{E}=\mathrm{a} \operatorname{mad}+\mathrm{b} \operatorname{mgd}+\mathrm{c} \text { cbd }+\mathrm{d} \text { enad }
$$




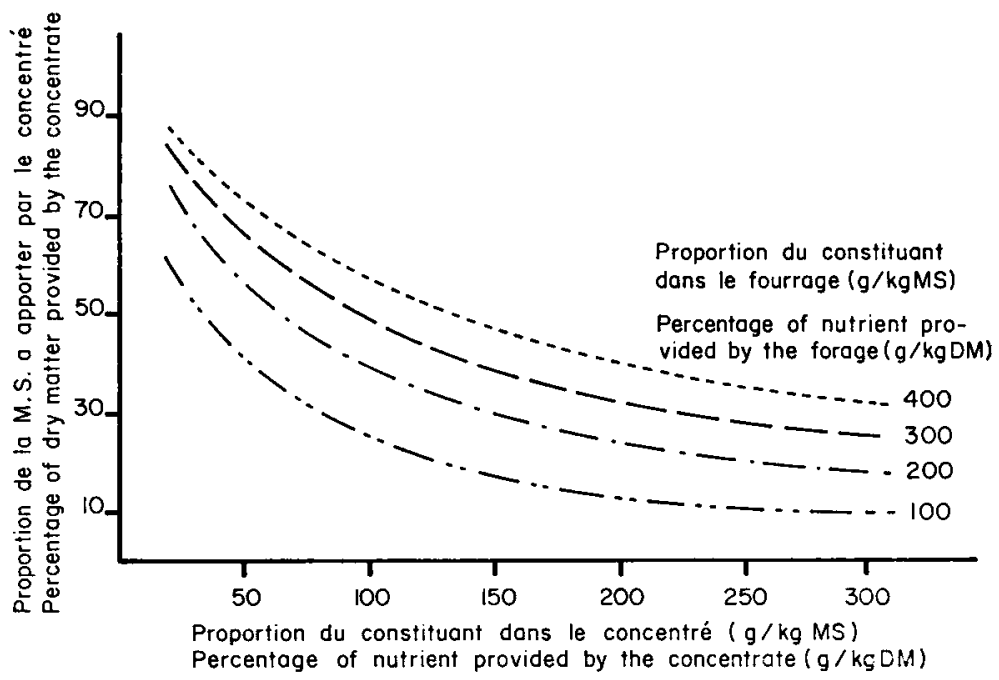

Fig. 13

Détermination de la proportion minimale d'aliment concentré

à apporter dans la matière sèche de la ration pour atteindre un degré donné de précision (1).

Determination of the minimal percentage of concentrate in the diet matter to obtain a given degree of accuracy (1).

(1) Cas où l'aliment concentré apporte au moins 25 p. 100 du constituant considéré dans la ration - Case where the concentrate provided at least 25 p. 100 of a given nutrient in the diet.

Si on suppose qu'il n'y a pas d'interaction digestive, l'incertitude exprimée en terme de variance de cette valeur énergétique liée à celle de la digestibilité est :

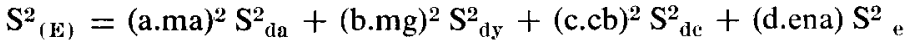

$$
\begin{aligned}
& S^{2}{ }_{(\mathbf{E})}=(\mathrm{a} . \mathrm{ma})^{2} \mathbf{S}^{2}{ }_{\mathrm{a}}+(\mathrm{b} . \mathrm{mg})^{2} \mathbf{S}^{2}{ }_{\mathrm{d} y}+(\mathrm{c} . \mathrm{cb})^{2} \mathbf{S}^{2}{ }_{\mathrm{dc}}+\text { (d.ena) } \mathbf{S}^{2}{ }_{\mathrm{de}}
\end{aligned}
$$

En raison des variations de composition des aliments concentrés, une même incertitude sur un coefficient de digestibilité aura des poids différents selon le composant considéré. En outre, l'adoption d'un fourrage unique pour estimer la valeur énergétique des aliments concentrés, aboutit à des précisions globales d'estimation différentes selon l'aliment considéré. Un rapide calcul montre ainsi, qu'indépendamment des phénomènes d'interaction digestive, l'emploi de foin de luzerne aboutit à une meilleure précision de l'estimation de la valeur énergétique pour du tourteau de soja que de l'orge. Dans un même ordre d'idée, il serait peut-être souhaitable de rechercher, pour chaque type de matière première ou d'aliment concentré, le fourrage le plus adapté pour obtenir une précision maximale. Ainsi, le foin de luzerne permet d'obtenir, à proportion comparable, des valeurs plus précises pour le CUD de l'ENA des céréales que l'ensilage de maïs.

La prise en compte des valeurs du CUD du foin seul, aboutit à des valeurs moyennes très comparables; cependant l'accroissement du nombre de résultats, en comparaison de la faible variabilité des valeurs de digestibilité du foin, a permis dans 
tous les cas de réduire sensiblement l'incertitude sur les valeurs des coefficients de digestibilité du concentré.

Avec l'emploi du système de pondération, les valeurs des coefficients de digestibilité ont été intermédiaires entre ceux obtenus par les méthodes des différences non pondérées et celles des équations et par régression. La pondération présente l'avantage de s'affranchir des variations d'incertitude liées à la proportion de l'élément apporté par l'aliment concentré ; ainsi elle a permis de fournir une information aussi précise sur la digestibilité des const:tuants pariétaux que sur celle des autres composants des aliments (cf. première composante principale, fig. 11). Lorsque le nombre de données est suffisant, il est utile de réaliser un histogramme des valeurs obtenues, en particulier pour les méthodes à foin variable ; l'allure de ce dernier peut en effet aider à déceler des phénomènes d'interaction digestive ou bien la présence de données très aberrantes.

\section{B. La méthode des équations}

La méthode des équations présente l'avantage de ne pas attribuer entièrement l'effet d'interaction à l'aliment concentré mais de le répartir entre les deux aliments utilisés. La généralisation de l'utilisation des ordinateurs permet d'envisager, par l'emploi d'un programme adapté(1), l'application systématique de cette méthode même lorsque le nombre d'observations est important. Ce programme permet non seulement de calculer toutes les combinaisons possibles mais aussi de sélectionner les combinaisons qui permettent d'atteindre la précision maximale.

\section{La méthode par régression}

Cette méthode a abouti à des résultats qui sont dans l'ensemble tout à fait comparables à la méthode des équations. La régression présente, en outre, l'avantage de pouvoir tester facilement l'existence de phénomènes d'interaction digestive ainsi que l'influence du niveau alimentaire. Ce dernier aspect est important car il permet, le cas échéant, d'extrapoler les valeurs mesurées de digestibilité pour le niveau d'entretien. Lorsque les coefficients de digestibilité d'un composant du fourrage et du concentré sont proches, moins de 5 points d'après nos résultats, la corrélation n'est plus significative.

Cependant, les résultats obtenus ont montré que la variation résiduelle de part et d'autre de la droite de régression gardait dans ce cas une signification par rapport au problème étudié. L'absence de linéarité n'a jamais eu d'influence spécifique significative avec les données considérées. Ce phénomène ne signifie cependant pas qu'il n'y a jamais eu d'interactions digestives, car la non-linéarité suppose une large plage de variations de la proportion $\mathrm{C}$ du principe apporté par le concentré et celle-ci a pu être insuffisante. Ainsi, la quatrième composante a mis en évidence l'existence d'interactions digestives qui se traduisent par des distorsions non négligeables des résultats de digestibilité entre les méthodes des différences d'une part, et celle des équations ou par régression d'autre part. Un test de comparaison du CUD du foin obtenu par régression avec celui mesuré quand le fourrage est distribué seul, permet, dans certains cas, et notamment si la plage de variation de $\mathrm{C}$ est trop faible, de mettre en évidence une inter-

(1) Les auteurs tiennent ce programme à la disposition des personnes qui pourraient être intéressées. 
action digestive ou un effet du niveau alimentaire. La valeur obtenue pour le CUD du concentré n'est alors valable que pour la plage de variation testée.

Entre les méthodes des équations et par régression qui fournissent des valeurs de CUD et des incertitudes sur ces valeurs comparables, il nous semble que la régression soit plus riche d'informations et plus facile à appliquer. En pratique, les résultats obtenus montrent qu'il est intéressant d'utiliser un programme de régression progressive ; cependant dans ce cas, il est nécessaire d'imposer comme première variable entrante, l'influence linéaire de la proportion $\mathrm{C}$ du composant apporté par l'aliment concentré. Il est en outre utile d'analyser la variation résiduelle de cet ajustement linéaire des données (normalité...). Pour un seul des cas considérés (digestibilité de la fraction NDFADF du régime riche) le niveau alimentaire a présenté une influence spécifique sur la digestibilité. Cependant, le niveau alimentaire a largement varié au cours des deux expérimentations : de 1,03 à 3,36 . Cette absence d'influence va dans le sens de certains résultats enregistrés par DOREAU \& REMOND (1982). Ces faits suggèrent que les variations des potentiels de production de lait, liés à des stades physiologiques ou à des animaux différents, sont associés à des variations parallèles du développement de la panse. Fell et al. (1972) ont d'ailleurs observé de telles variations dans le cas de brebis en début de lactation. Ces résultats ne permettent donc pas d'appliquer à des animaux à production élevée, présentant de ce fait des niveaux alimentaires nettement supérieurs à ceux de l'entretien, des relations entre la digestibilité et le niveau alimentaire obtenus sur des animaux placés à des niveaux relativement proches de l'entretien.

\section{Conclusion}

\section{Choix d'une méthode de travail}

Ce travail apporte une contribution à la mise au point d'une méthode normalisée de calcul des coefficients de digestibilité des aliments concentrés. Il a permis de souligner l'intérêt de confronter les résultats issus des trois familles de méthodes : différence, régression, équation.

Dans la recherche de la valeur d'un CUD, le problème majeur est de savoir s'il vaut mieux chercher à focaliser le niveau des apports de concentrés autour d'une valeur la plus élevée possible et d'interpréter par une des méthodes des différences, ou bien s'il est préférable de concevoir des expérimentations à taux d'apports très divers et de les interpréter avec la méthode par régression. Lorsqu'il y a une interaction digestive, la seconde méthode de travail peut seule permettre de la démontrer. Lorsqu'il n'y a pas d'interaction, ce dont on ne peut être certain a priori, la seconde approche associée à une interprétation par régression semble encore être préférable en raison de la richesse des résultats qu'elle founit.

La méthode des équations, qui dans le cas présent fournit des résultats proches de cette dernière, n'est pas à conseiller dans la pratique car elle nécessite de faire beaucoup de calculs et, comme la méthode des différences, ne permet pas de mettre clairement en évidence des éventuels effets dus à une interaction digestive ou au niveau alimentaire. 
En définitive, la meilleure méthode de calcul du CUD d'un aliment concentré est la méthode par régression appliquée à des niveaux variables d'apports du concentré. En outre, il semble que la méthode des équations soit préférable aux méthodes procédant par différence.

Accepté pour publication en avril 1982.

\section{Summary \\ Comparison of different methods for evaluation of digestibility coefficients of concentrate feeds in ruminants}

Digestibility of a ruminant compound feed cannot be directly measured. Various methods have been proposed to evaluate the digestibility coefficients in such a situation and the aim of the present work was to compare systematically these main methods. The data used were derived from measurements obtained with 4 mixed diets (roughage + concentrate) and took into account 16 components (tabl. 1, 2,3).

The classical method, or "difference method», gives high unreliable results when the percentage of the component provided by the concentrate in the diet was less than 20 p. 100 (fig. 2). The use of ponderated values did not modify statistically the digestibility coefficients values, but allowed to reduce the safety ranges (fig. 3). Similar conclusions were obtained when individual variations of the roughage digestibility were taken into account instead of a sole mean value ("variable hay» difference method) (fig. 4).

With the equation-method there was a minimal value of the incertitude which is a function of the number of eliminated data and the value of the minimal difference allowed between two levels of concentrate incorporation (fig. 9). This mean level was about 10 p. 100. A stepwise multiple regression model including the level of concentrate was also used to test phenomena of associative digestibilities, the influence of the feeding level and various interactions.

Results obtained with the equation and regression methods were rather similar. They were not so highly correlated with those provided by the difference method (tabl. 5).

Comparison of these various methods indicated that the reliability is largely influenced by the mean concentrate percentage, but also by the variations of these percentages. The regression method seemed to be the most suited because its possibility to test simultaneously the influence of different exogenous parameters (fig. 11 and 12).

The present study emphasizes the importance of knowing, the specific reliability and the method used to determine each published digestibility coefficient.

\section{Références bibliographiques}

Charlet-Lery G., Leroy A.M., 1955. Etude comparée de la digestibilité du son de froment par diverses méthodes. Ann. Zootech., 4, 111-120.

DOREAU M., REMOND B., 1982. Comportement alimentaire et utilisation digestive d'une ration de composition constante chez la vache laitière en fin de gestation et début de lactation. Reprod. Nurr. Dévelop., 22, 307-324.

Fell B.F., Campbell R.A., Mackie W.S., Weekes T.E.C., 1972. Changes associated with pregnancy and lactation in some extra reproductive organs of the ewe. J, agric. Sci., 7S, 397-407.

Frederiksen J.H., 1973. Method studies relating to digestibility trials with ruminants. Acta A gric. Scand., 23, 17-33. 
Gasnier A., Vachel J.P.. 1952. Recherches sur la méthode de mesure de la digestibilité d'un aliment. Condition d'emploi de cette méthode. Ann. Zootech., 1, 157-174.

Giger S., Dorleans M., Sauvant D., 1983. Adaptation of the Van Soest method to a routine determination of concentrate feedstuffs (à paraître).

Giger S., Sauvant D., Dorleans M., Hervieu J., 1983 a. Comparaison de la valeur nutritive de deux aliments concentrés de mêmes caractéristiques analytiques classiques. Ann. Zootech. (à paraître).

Giger S., Sauvant D., Dorleans M., Hervieu J., 1983 b. Variations possibles de la teneur en paroi de deux aliments concentrés de même digestibilité de la matière organique. Ann. Zootech. (à paraître).

KNieriem K. Von, 1900. Der Roggen als Kraftfuttermittel. Landw. Jahrb., 29, 489, cité par SCHNEIDER et Flatt, 1975.

Mitchell H.H., 1937. The importance of the relations between energy, protein and minerals. in measuring the nutritive value of feeds and rations. Am. Soc. anim. Prod. Soc., 30, 29.

Sauvant D., Giger S., 1980. Variations de la teneur en composants de la paroi végétale pour des mélanges alimentaires de même valeur nutritive. $31^{\circ}$ Réunion F.E.Z., Commission de nutrition, session 5, 1-4 septembre 1980.

Schieman R., Nehring K., Hoffman L., Jentsch W., Chudy A., 1971. Energetische Futterbewertung und Energienormen. VEB Deutscher Landwirtschaftverlag, Berlin.

SChNeIder B.H., FlatT W.P., 1975. Digestibility by difference. In : The evaluation of feeds through digestibility experiments, 151-163. Ed. by the University of Georgia Press, Athens.

VAN Soest P.J., 1963. Use of detergents in the analysis of fibrous feeds. II. Rapid method for the determination of fibre and lignin. J.A.O.A.C., 46, 829-835.

VAN Soest P.J, WINe R.H., 1967. Use of detergents in the analysis of fibrous feeds. IV. Determination of plant cell wall constituents J.A.O.A.C., 50, 50-55. 